\title{
SÍNTESE DE 2-IODOBENZAMIDAS E 3-(IODOACETAMIDO)BENZAMIDAS LIGADAS À D-GALACTOSE E SUAS REAÇÕES DE CARBOCICLIZAÇÃO RADICALAR MEDIADAS POR HIDRETO DE TRI- $N$-BUTILESTANHO
}

\author{
Daniel Henriques Soares Leal, Carla Graziella Queiroga, Magno Carvalho Pires, Maria Auxiliadôra Fontes Prado e \\ Ricardo José Alves* \\ Departamento de Produtos Farmacêuticos, Faculdade de Farmácia, Universidade Federal de Minas Gerais, Av. Presidente Antônio \\ Carlos, 6627, 31270-901 Belo Horizonte - MG, Brasil \\ Amary Cesar \\ Departamento de Química, Instituto de Ciências Exatas, Universidade Federal de Minas Gerais, Av. Presidente Antônio Carlos, \\ 6627, 31270-901 Belo Horizonte - MG, Brasil
}

Recebido em 21/1/09; aceito em 26/5/09; publicado na web em 30/10/09

\begin{abstract}
SYNTHESIS OF 2-IODOBENZAMIDES AND 3-(IODOACETAMIDO)BENZAMIDES LINKED TO D-GALACTOSE AND THEIR TRI- $N$-BUTYLTIN HYDRIDE-MEDIATED RADICAL CARBOCYCLIZATION REACTIONS. Starting from methyl 6-O-allyl4-azido-2,3-di- $O$-benzyl-4-deoxy- $\alpha$-D-galactopyranoside, four new derivatives containing 2-iodobenzamido and 3-(iodoacetamido) benzamido groups were synthesized. These four compounds were submitted to tri- $n$-butyltin hydride mediated radical cyclization reactions, resulting in two macrolactams from 11- and 15-endo aryl radical cyclization. The corresponding four hydrogenolysis products were also obtained. The structures of the new compounds were elucidated by ${ }^{1} \mathrm{H}$ and ${ }^{13} \mathrm{C}$ NMR spectroscopy, DEPT, COSY, HMQC and HMBC experiments.
\end{abstract}

Keywords: aryl radical cyclization; macrolactam; D-galactose derivatives.

\section{INTRODUÇÃO}

Os macrociclos, produtos naturais que apresentam grande variedade estrutural, constituem uma importante classe de compostos, devido às suas diversas e relevantes atividades biológicas e ao fato de muitos deles serem usados como fármacos. Neste contexto citam-se, a título de exemplo, a eritromicina (antibacteriano), a anfotericina B (antifúngico) e o tacrolimus (imunomodulador). ${ }^{1-3}$

Tendo em vista a importância dos macrociclos, muitos métodos para síntese desse tipo de substância foram e vêm sendo desenvolvidos e relatados. Essencialmente, os macrociclos podem ser obtidos por ciclização de precursores de cadeia longa ou por clivagem de ligações internas em sistemas policíclicos. Estes métodos podem variar de acordo com o tipo de ligação formada ou rompida $(\mathrm{C}=\mathrm{C}, \mathrm{C}-\mathrm{C}, \mathrm{C}-\mathrm{O}$ etc) e também com a natureza eletrônica da ligação a ser formada ou rompida (iônicos ou radicalares). ${ }^{4}$ Um dos processos de formação de macrociclos consiste na adição intramolecular de radicais a ligações múltiplas a partir de precursores contendo um halogênio (normalmente iodo ou bromo) e uma insaturação. A formação dos radicais envolve reação com o hidreto de tri- $n$-butilestanho, e a adição pode ocorrer na posição externa (ciclização endo) ou interna (ciclização exo) da insaturação (Figura 1)..$^{5-23}$

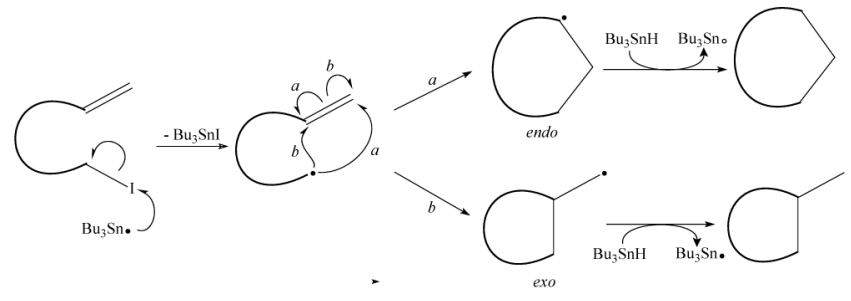

Figura 1. Possíveis modos de ciclização na reação de carbociclização radicalar mediada por $\mathrm{Bu}_{3} \mathrm{SnH}$

*e-mail: ricardodylan@farmacia.ufmg.br
Considerando a importância dos macrociclos e o desafio que constituem as reações de macrociclização, ${ }^{2}$ um programa de síntese de macrociclos por meio de reações de carbociclização radicalar mediadas por hidreto de tri- $n$-butilestanho tem sido desenvolvido, desde 1996, no Laboratório de Química Farmacêutica da Faculdade de Farmácia da UFMG, em colaboração com o Departamento de Química do ICEx/UFMG. No âmbito deste programa, vários e diferentes substratos já foram submetidos às condições de carbociclização radicalar com hidreto de tri- $n$-butilestanho $0^{24-33} \mathrm{e}$ foram isolados vários produtos de carbociclização radicalar. ${ }^{24-31,33}$ As estruturas de alguns destes substratos e seus produtos de ciclização e de hidrogenólise, com os respectivos rendimentos, encontram-se na Tabela 1.

A análise conjunta dos resultados até então obtidos indica que a presença da unidade sacarídica favoreceu as reações de carbociclização, mas não interferiu no modo de ciclização, uma vez que, em todos os casos, foram isolados apenas os produtos provenientes de ciclização pelo modo endo, ${ }^{24,25,31} \mathrm{em}$ acordo com o que se encontra descrito na literatura. ${ }^{6-8,12,34-39}$ Além disso, os resultados demonstraram o potencial das reações radicalares mediadas por $\mathrm{Bu}_{3} \mathrm{SnH}$ para a obtenção de compostos cíclicos a partir de substratos do tipo 2-iodobenzamidas contendo um grupo aliloxila na cadeia lateral..$^{24,25,31}$

Dando continuidade ao programa, foi proposta para este trabalho a síntese de quatro substâncias de configuração D-galacto $(\mathbf{1 3}, \mathbf{1 4}, 19$ e 20) que seriam submetidas às condições de ciclização radicalar. As 2-iodobenzamidas 13, alílica, e 14, cinamílica, poderiam conduzir às benzomacrolactamas 11-endo 15 e 16 e/ou 10 -exo 17 e 18, e as 3-(iodoacetamido)benzamidas 19, alílica, e 20, cinamílica, poderiam conduzir às benzomacrolactamas 15-endo 21 e 22 e/ou 14-exo 23 e 24 (Figura 2).

Outra vertente de trabalhos realizados pelo grupo consistiu na síntese de análogos cinamílicos das benzamidas 1, 7 e 10 (Tabela 1), respectivamente, 25, 26 e 27 (Figura 3). Nas reações de carbociclização com essas substâncias não foram isolados produtos de ciclização e sim os produtos de hidrogenólise correspondentes e um derivado de substituição do átomo de iodo pelo grupo tri- $n$-butilestanila. ${ }^{32,33} \mathrm{~A}$ 
Tabela 1. Substratos, produtos e seus respectivos rendimentos nas reações das 2-iodobenzamidas 1, 4, 7 e $10 \mathrm{com} \mathrm{Bu}_{3} \mathrm{SnH}$

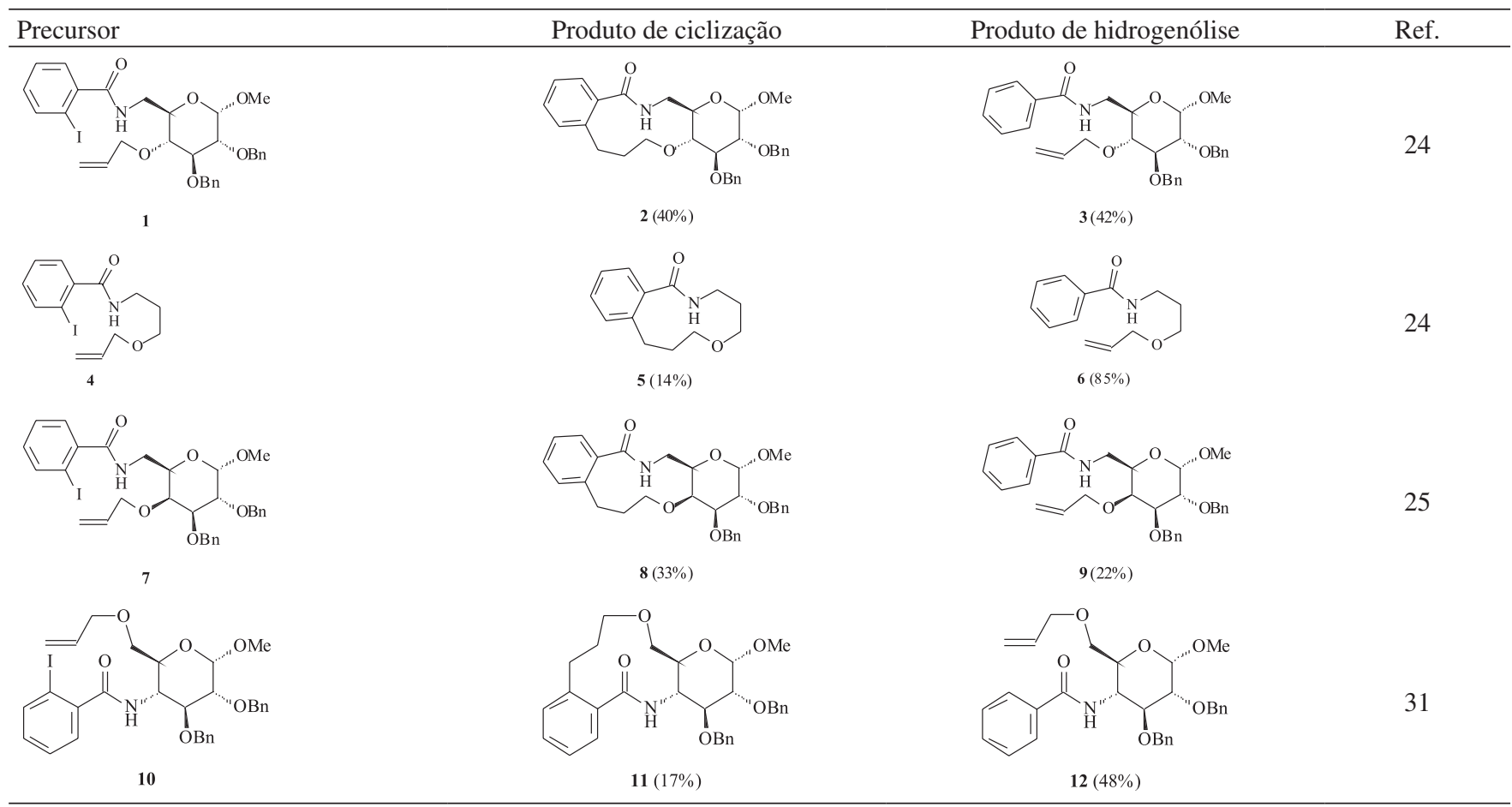
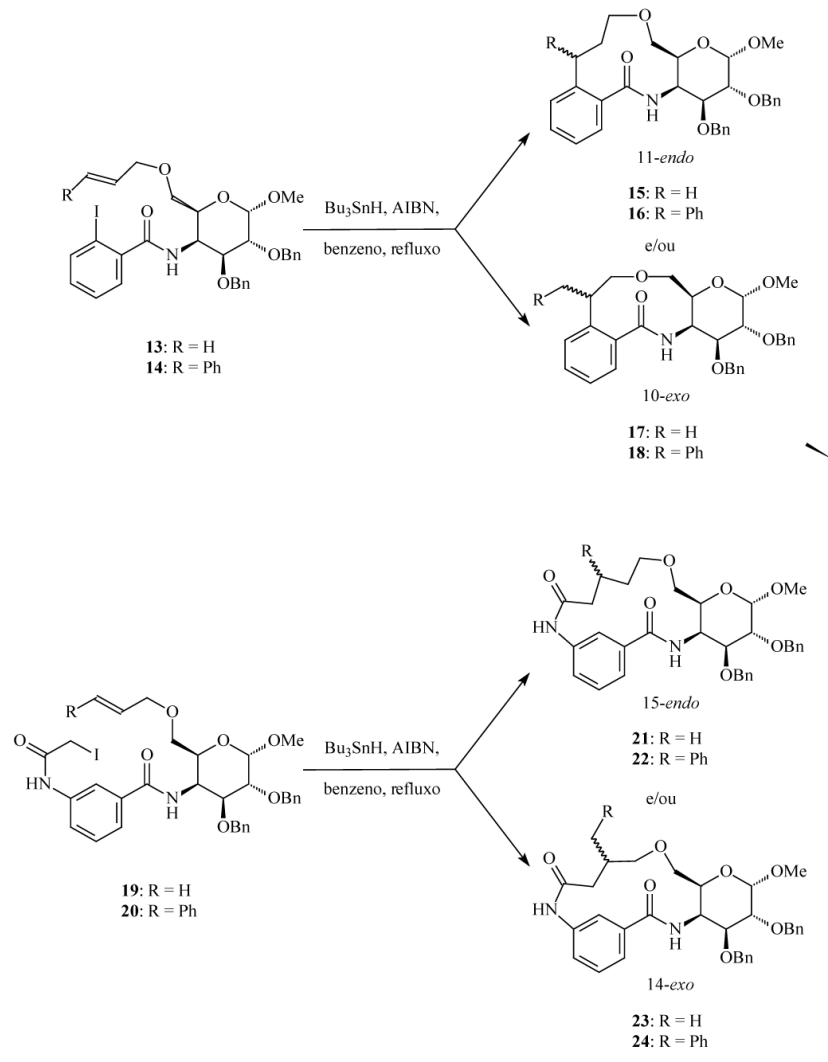

Figura 2. Benzomacrolactamas possíveis de serem formadas nas reações de ciclização radicalar das benzamidas 13, 14, 19 e $20 \mathrm{com} \mathrm{Bu}_{3} \mathrm{SnH}$

benzamida 14 (Figura 2), objeto de síntese deste trabalho, é regioisômero e epímero de $\mathbf{2 6}$ e 27 (Figura 3), respectivamente.

As 3-(iodoacetamido)benzamidas 19 e 20 (Figura 2) foram concebidas devido à possibilidade de formação de produtos cíclicos de tamanho estendido (15-endo ou 14-exo) em relação aos até então obtidos ou planejados nos trabalhos do grupo. Segundo dados da literatura, ${ }^{7,12}$ a formação de anéis maiores é mais favorecida em relação aos de tamanho médio (oito a doze membros).

Além de se obter as macrolactamas inéditas, teve-se como objetivo neste trabalho comparar os resultados das reações de carbociclização radicalar das benzamidas $\mathbf{1 3}$ e $\mathbf{1 4}$ (Figura 2) com aqueles observados em trabalhos anteriores ${ }^{24,25,31-33}$ (Tabela 1 e Figura 3) para se verificar ou confirmar as relações entre as estruturas químicas dos precursores $\mathrm{e}$ as reações mediadas por $\mathrm{Bu}_{3} \mathrm{SnH}$. Assim, a comparação dos resultados das reações de $\mathbf{1 3}$ (Figura 2) com seu regioisômero 7 (Tabela 1) e de 14 (Figura 2) com 26 (Figura 3) permitiria verificar se a localização dos dois grupos envolvidos na ciclização (2-iodobenzamido e aliloxila ou cinamiloxila) em C-4 ou C-6 do anel piranosídico influenciaria a ocorrência, a extensão e o modo de ciclização. Da mesma forma, seria possível observar a influência da estereoquímica de C-4, por comparação dos resultados das reações de 13 e 14 (Figura 2) com seus respectivos epímeros de configuração glico $\mathbf{1 0}$ (Tabela 1) e 27 (Figura 3). Além disso, a comparação das reações das benzamidas 13 com 14 (Figura 2) permitiria confirmar se, também nessa série, a substituição do grupo $O$-alila por $O$-cinamila prejudicaria a reação de ciclização, como se observara em trabalhos anteriores. ${ }^{32,33}$

\section{RESULTADOS E DISCUSSÃO}

Para a síntese das benzamidas $\mathbf{1 3}, \mathbf{1 4}, \mathbf{1 9}$ e 20, foi planejada a rota mostrada na Figura 4.

O azidoálcool 28, já descrito na literatura, ${ }^{40}$ foi obtido em sete etapas a partir do $\alpha$-D-glicopiranosídeo de metila: proteção das hidroxilas de C-4 e C-6 como acetal benzilidênico ${ }^{41}$ benzilação das hidroxilas de C-2 e C- $3,{ }^{42}$ remoção do grupo acetal benzilidênico, ${ }^{43}$ mesilação das hidroxilas de C-4 e C- $6,{ }^{44}$ substituição regiosseletiva do grupo mesila de C-6 por benzoíla, ${ }^{40}$ substituição do grupo mesila de C-4 pelo grupo azido ${ }^{45}$ e hidrólise do grupo benzoíla. ${ }^{40} \mathrm{O}$ derivado 28 foi obtido com um rendimento global de $66 \%$ a partir do produto da terceira etapa da rota de síntese descrita (2,3-di- $O$-benzil- $\alpha$-D-glicopiranosídeo 
de metila). O rendimento de $\mathbf{2 8}$ descrito na literatura, ${ }^{40}$ utilizando-se o 2,3-di- $O$-benzil- $\alpha$-D-glicopiranosídeo de metila como material de partida foi de $43 \%$. A partir de $\mathbf{2 8}$, as 2-iodobenzamidas 13 e 14 foram obtidas em três etapas, e as 3-(iodoacetamido)benzamidas $\mathbf{1 9}$ e 20 em quatro etapas.

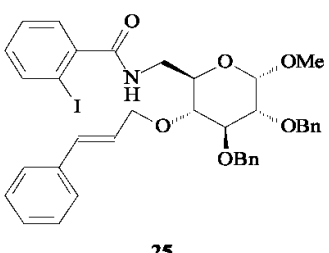

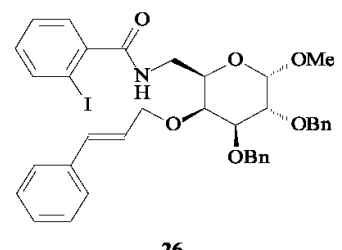

26

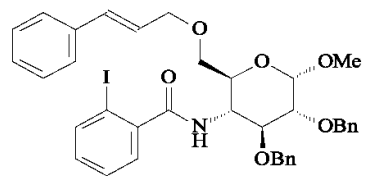

27

Figura 3. Estruturas de 25, 26 e 27

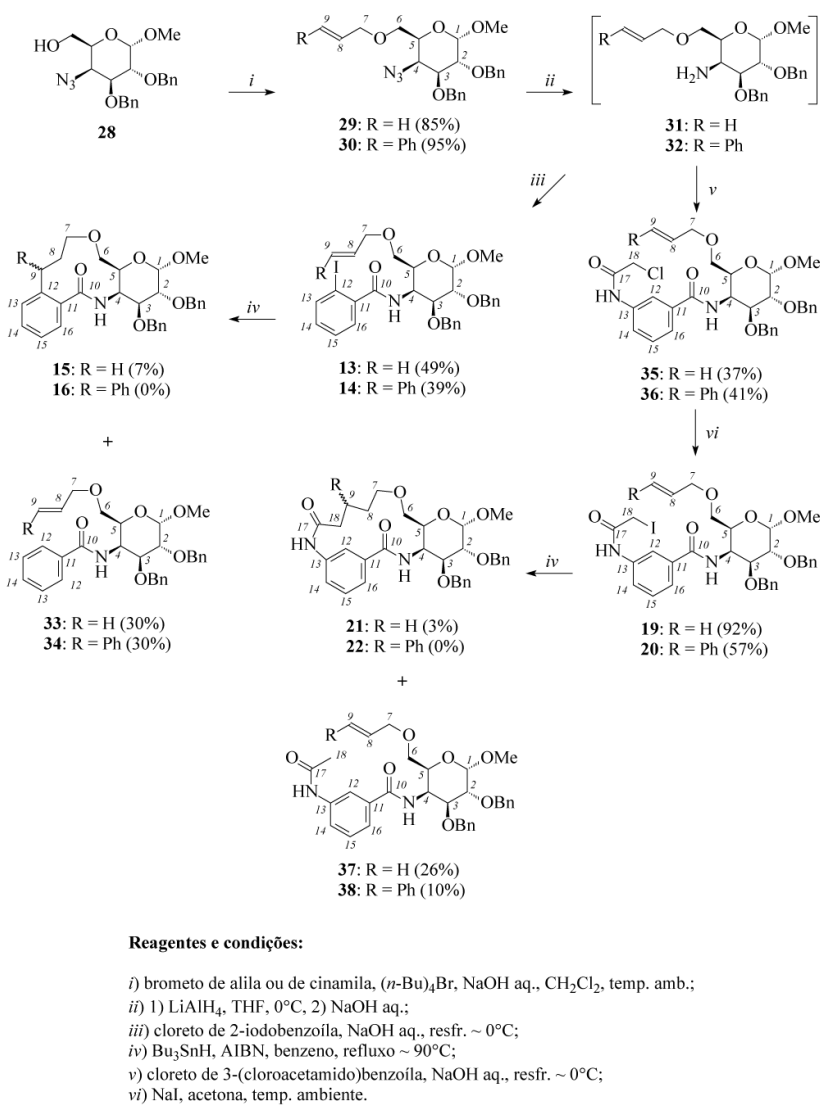

Figura 4. Rota sintética utilizada na obtenção das benzamidas 13, 14, 19 e 20 e dos produtos isolados das reações de carbociclização radicalar com $\mathrm{Bu}_{3} \mathrm{SnH}$

O azidoálcool 28 foi, por reação com brometos de alila e cinamila, respectivamente, em condições de transferência de fase, ${ }^{42}$ convertido nos éteres $\mathbf{2 9}$ ( $85 \%$ de rendimento) e $\mathbf{3 0}$ (95\% de rendimento) que, por redução dos grupos azido com hidreto de alumínio e lítio, ${ }^{46}$ levaram às aminas 31 e 32, utilizadas sem purificação adicional diretamente na etapa seguinte. Por reação das aminas com o cloreto de 2 -iodoben- zoíla, ${ }^{47,48}$ foram obtidas as 2-iodobenzamidas 13 (49\%) e 14 (39\%). A reação das aminas $\mathbf{3 1}$ e $\mathbf{3 2}$ com o cloreto de 3-(cloroacetamido) benzoíla ${ }^{47-49}$ levou às 3-(cloroacetamido)benzamidas $\mathbf{3 5}$ (37\%) e $\mathbf{3 6}$ $(41 \%)$. Por substituição nucleofílica do átomo de cloro por iodo, ${ }^{50}$ foram obtidas as 3-(iodoacetamido)benzamidas $\mathbf{1 9}(92 \%)$ e $20(57 \%)$. Cada uma das benzamidas 13, 14, 19 e 20 (Figura 2) foi submetida à reação de ciclização radicalar nas condições preconizadas, de modo a minimizar a formação do produto de hidrogenólise e a ocorrência de reações intermoleculares: alta diluição e lenta adição da solução de $\mathrm{Bu}_{3} \mathrm{SnH}$ e quantidade catalítica de AIBN em benzeno sobre a solução das benzamidas em benzeno sob refluxo. ${ }^{12,51,52}$ Para otimização dos rendimentos, as condições de reação foram variadas para as benzamidas 13, 14 e 19. Para a benzamida 20, foi realizada apenas uma tentativa. As condições empregadas e os resultados obtidos encontram-se na Tabela 2.

Após remoção do solvente, os resíduos obtidos foram purificados por cromatografia em coluna de sílica. Para as benzamidas alílicas 13 e 19, foram isolados, em cada caso, dois produtos, identificados como sendo as macrolactamas provenientes de ciclização 11-endo $\mathbf{1 5}$ (7\%, máximo) e 15-endo 21 (3\%, máximo), além de seus respectivos produtos de hidrogenólise $\mathbf{3 3}$ (30\%) e 37 (26\%). Das reações das benzamidas cinamílicas $\mathbf{1 4}$ e 20, foram isolados apenas seus correspondentes produtos de hidrogenólise 34 (30\%, máximo) e 38 (10\%) (Figura 4).

A comparação do rendimento de $\mathbf{1 5}$ (7\%), produto de ciclização da benzamida $\mathbf{1 3}$, com resultados anteriores $\mathbf{- 1}$ levando a $\mathbf{2}(40 \%)$, 7 levando a $8(33 \%)$ e $\mathbf{1 0}$ que conduziu a $\mathbf{1 1}(17 \%)$ - (Tabela 1), reforça a observação feita em trabalho anterior ${ }^{31}$ de que a ciclização é mais favorecida quando os grupos $O$-alila e 2-iodobenzamida estão localizados, respectivamente, nas posições 4 e 6 do anel piranosídico. Uma possível explicação para esta diferença estaria na maior possibilidade de formação de ligação de hidrogênio intramolecular entre o hidrogênio amídico e o oxigênio alílico nas benzamidas 1 e 7 do que nas benzamidas $\mathbf{1 0}$ e $\mathbf{1 3}$ e, por consequência, nos correspondentes radicais arila. Esta possibilidade foi investigada com cálculos teóricos nos níveis PM3 e DFT/B3LYP, ainda não publicados, os quais parecem corroborar esta hipótese. ${ }^{53}$

Com base nos deslocamentos químicos e nas constantes de acoplamento escalar entre os hidrogênios amídicos e os hidrogênios dos carbonos da posição 4 dos anéis piranosídicos (posição secundária) em 10 e 13 e nos correspondentes produtos de ciclização e hidrogenólise calculados nos espectros de $\mathrm{RMN}{ }^{1} \mathrm{H}$ (Tabela 3), e de acordo com a correlação de Karplus, estes hidrogênios se encontrariam, provavelmente, em relação antiperiplanar (ângulo torsional $\mathrm{H}$ N-C-4-H-4 em torno de $\left.180^{\circ}\right),{ }^{54}$ do que se depreende a existência de restrição conformacional ao giro livre da ligação N-C-4. Com relação ao grupo $O$-alila, esse teria um grau de liberdade conformacional a mais em C-6 do que quando em C-4.

Em 10 e 13, portanto, a formação da referida ligação de hidrogênio intramolecular seria prejudicada (Figura 5), permitindo um maior número de conformações dos radicais arila em que a cadeia alílica se encontra mais afastada da cadeia benzamídica, correspondendo a um menor número de conformações favoráveis à ciclização. Em 1 e 7, como a cadeia benzamídica se encontra na posição 6 do anel piranosídico (posição primária), a ligação N-C-6 poderia girar com maior liberdade, além da possibilidade de giro livre da ligação $\mathrm{C}$ 5-C-6. Com um grau de liberdade conformacional a mais (a ligação C-5-C-6) e com menor restrição ao giro da ligação N-C-6, haveria assim, em 1 e 7, maior número de conformações favoráveis à ciclização, uma vez que a ligação de hidrogênio poderia ser formada com maior probabilidade, mantendo as cadeias laterais mais próximas (Figura 5). Isto poderia explicar o motivo de as reações de ciclização funcionarem melhor quando o grupo 2-iodobenzamido está em C-6 (1 e 7) do que quando está em C-4 (10 e 13). 
Tabela 2. Condições empregadas e resultados obtidos nas reações de carbocilização radicalar das benzamidas $\mathbf{1 3}, \mathbf{1 4}, \mathbf{1 9}$ e $\mathbf{2 0}$

\begin{tabular}{|c|c|c|c|c|}
\hline $\begin{array}{l}\text { Precursor, } \\
\text { reação, quant. }\end{array}$ & Conc. benzamida & Conc. $\mathrm{Bu}_{3} \mathrm{SnH}$ & $\begin{array}{l}\text { Prod. ciclização, } \\
\text { quant., rendimento }\end{array}$ & $\begin{array}{l}\text { Prod. hidrogenólise, } \\
\text { quant., rendimento }\end{array}$ \\
\hline $\mathbf{1 3}, 1^{\mathrm{a}}, 0,37 \mathrm{mmol}$ & 7,6 mmol/L & $11,4 \mathrm{mmol} / \mathrm{L}$ & $\mathbf{1 5}, 6,6 \mathrm{mmol}, 1,8 \%$ & $\mathbf{3 3}, 74 \mathrm{mmol}, 20 \%$ \\
\hline $\mathbf{1 3}, 2^{\mathrm{a}}, 0,33 \mathrm{mmol}$ & $8,0 \mathrm{mmol} / \mathrm{L}$ & $12,0 \mathrm{mmol} / \mathrm{L}$ & $\mathbf{1 5}, 19 \mathrm{mmol}, 5,6 \%$ & 33, $0,16 \mathrm{mmol}, 49 \%$ \\
\hline $\mathbf{1 3}, 3^{\mathrm{a}}, 0,32 \mathrm{mmol}$ & $5,3 \mathrm{mmol} / \mathrm{L}$ & $7,8 \mathrm{mmol} / \mathrm{L}$ & $\mathbf{1 5}, 23 \mathrm{mmol}, 7,4 \%$ & 33, $96 \mathrm{mmol}, 30 \%$ \\
\hline $\mathbf{1 4}, 1^{\mathrm{a}}, 0,56 \mathrm{mmol}$ & 7,5 mmol/L & $12,3 \mathrm{mmol} / \mathrm{L}$ & - & $\mathbf{3 4}, 0,17 \mathrm{mmol}, 30 \%$ \\
\hline $\mathbf{1 4}, 2^{\mathrm{a}}, 0,92 \mathrm{mmol}$ & $4,9 \mathrm{mmol} / \mathrm{L}$ & $8,6 \mathrm{mmol} / \mathrm{L}$ & - & $34,0,24 \mathrm{mmol}, 26 \%$ \\
\hline 19, $1^{\mathrm{a}}, 0,29 \mathrm{mmol}$ & $8,3 \mathrm{mmol} / \mathrm{L}$ & $14,9 \mathrm{mmol} / \mathrm{L}$ & $\mathbf{2 1}, 6,3 \mathrm{mmol}, 2,2 \%$ & $37,0,12 \mathrm{mmol}, 44 \%$ \\
\hline $\mathbf{1 9}, 2^{\mathrm{a}}, 0,10 \mathrm{mmol}$ & $4,0 \mathrm{mmol} / \mathrm{L}$ & $7,6 \mathrm{mmol} / \mathrm{L}$ & $\mathbf{2 1}, 3,0 \mathrm{mmol}, 3,0 \%$ & $37,26 \mathrm{mmol}, 26 \%$ \\
\hline $\mathbf{2 0}, 1^{\mathrm{a}}, 0,18 \mathrm{mmol}$ & $4,0 \mathrm{mmol} / \mathrm{L}$ & $6,7 \mathrm{mmol} / \mathrm{L}$ & - & $\mathbf{3 8}, 18 \mathrm{mmol}, 10 \%$ \\
\hline
\end{tabular}

Tabela 3. Dados de $\mathrm{RMN}^{1} \mathrm{H}\left(\delta\right.$, multiplicidade $\left.\mathrm{e}^{3} J\right)$ dos hidrogênios NH e H-4 das benzamidas precursoras $13,14,19$ e 20, dos produtos de ciclização 15 e 21 e dos produtos de hidrogenólise $33,34,37$ e 38

\begin{tabular}{lccc}
\hline Substância & $\mathrm{NH}, \boldsymbol{\delta}(\mathrm{ppm})$ & $\mathrm{H}-4, \delta(\mathrm{ppm})$ & ${ }^{3} J_{\mathrm{NH}-\mathrm{H} 4}(\mathrm{~Hz})$ \\
\hline $\mathbf{1 3}$ (benzamida) & $5,92(\mathrm{~d})$ & $4,95(\mathrm{~m})$ & $9,1 \mathrm{~Hz}$ \\
$\mathbf{1 5}$ (prod. ciclização) & $6,03(\mathrm{~d})$ & $5,07(\mathrm{ddd})$ & $10,4 \mathrm{~Hz}$ \\
$\mathbf{3 3}$ (prod. hidrogenólise) & $6,22(\mathrm{~d})$ & $5,00-4,95(\mathrm{~m})$ & $10,3 \mathrm{~Hz}$ \\
$\mathbf{1 4}$ (benzamida) & $5,91(\mathrm{~d})$ & $4,93(\mathrm{~m})$ & $10,0 \mathrm{~Hz}$ \\
$\mathbf{3 4}$ (prod. hidrogenólise) & $6,29-6,17(\mathrm{~m})$ & $5,00-4,95(\mathrm{~m})$ & - \\
$\mathbf{1 9}$ (benzamida) & $6,56(\mathrm{~d})$ & $4,99(\mathrm{dd})$ & $9,8 \mathrm{~Hz}$ \\
$\mathbf{2 1}$ (prod. ciclização) & $6,46(\mathrm{~d})$ & $5,04(\mathrm{dd})$ & $10,2 \mathrm{~Hz}$ \\
$\mathbf{3 7}$ (prod. hidrogenólise) & $6,30(\mathrm{~d})$ & $4,94(\mathrm{dd})$ & $9,7 \mathrm{~Hz}$ \\
$\mathbf{2 0}$ (benzamida) & $6,56-6,48(\mathrm{~m})$ & $5,02(\mathrm{dd})$ & $9,3 \mathrm{~Hz}$ \\
$\mathbf{3 8}$ (prod. hidrogenólise) & $6,31(\mathrm{~d})$ & $4,98(\mathrm{ddd})$ & $9,6 \mathrm{~Hz}$ \\
\hline
\end{tabular}

Já em relação à estereoquímica do carboidrato, observa-se que, com a configuração D-glico nas 2-iodobenzamidas $\mathbf{1}$ e 10, os rendimentos são melhores do que com a configuração D-galacto em 7 e 13 (42 e 17\% versus 33 e 7\%, respectivamente). Poder-se-ia atribuir esta diferença a um fator estérico, já que, nas 2-iodobenzamidas 7 e 13, há um dos grupos que participa da reação de ciclização, o de C-4, em posição axial no anel piranosídico $(O$-alila em 7 e 2 -iodobenzamido em 13), o que poderia desfavorecer a aproximação entre os grupos para que ocorra a ciclização. Em 1 e 10, os grupos estão em posição equatorial, na qual há menor efeito estérico, o que permite melhor aproximação dos grupos envolvidos na ciclização.

Confirmou-se, mais uma vez, a preferência pelo modo de ciclização endo em relação ao exo, como já estabelecido anteriormente. . $^{6-8,12,24,25,31}$

Confirmou-se também a impossibilidade, até o momento, da obtenção de produtos de ciclização de benzamidas contendo o grupo cinamila em substituição ao grupo alila. ${ }^{32,33} \mathrm{~A}$ ausência de produtos de ciclização a partir de derivados cinamílicos foi atribuída por Oliveira e colaboradores ${ }^{32}$ ao impedimento estérico causado pelo grupo fenila, que impediria a aproximação do radical arila ao carbono mais externo da ligação dupla para levar ao produto de ciclização formado pelo modo preferencial (endo). Outra hipótese aventada foi a de que haveria transferência de hidrogênio do grupo metilênico do grupo cinamila para o radical arila com formação de um radical alílico estável, que posteriormente levaria ao produto de hidrogenólise. ${ }^{32}$ De fato, resultados teóricos, usando cálculos no nível DFT ainda não publicados, sugerem que a causa do insucesso na obtenção de produ-

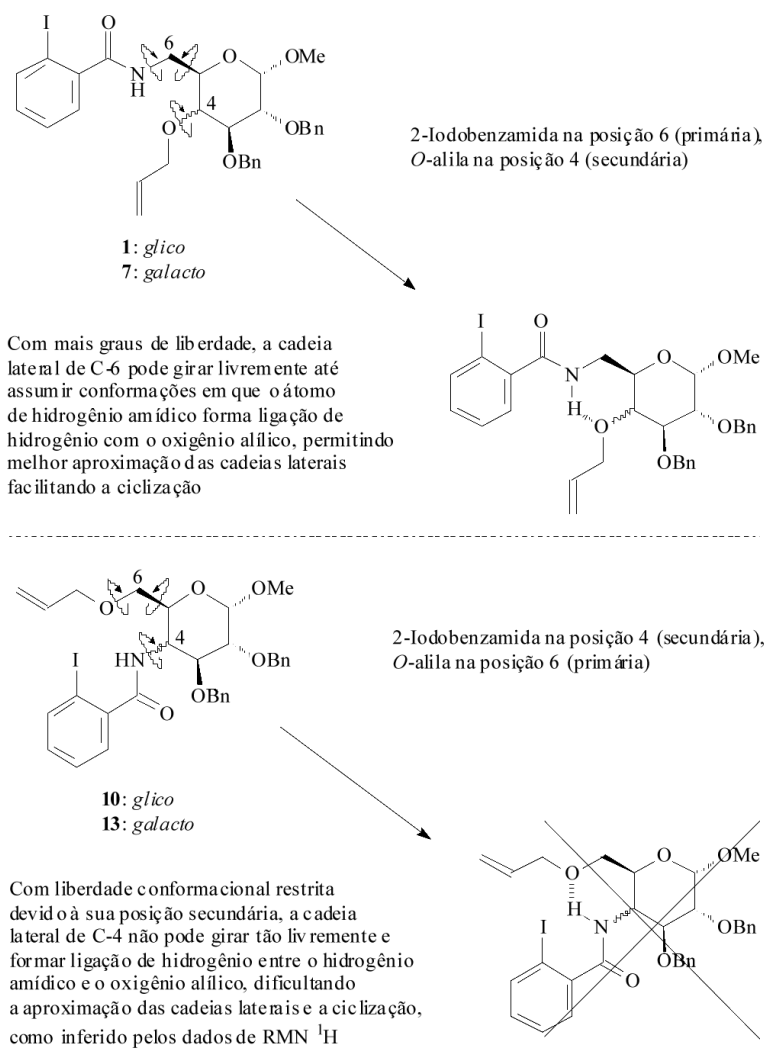

Figura 5. Aspectos conformacionais das benzamidas 1 e 7 e dos regioisômeros correspondentes 10 e 13

tos de ciclização cinamílicos se encontra, provavelmente, na maior acidez relativa dos hidrogênios metilênicos alifáticos cinamílicos em relação aos alílicos, cujos radicais resultantes da abstração de um desses átomos de hidrogênio poderiam levar a reações indesejáveis que inviabilizam as reações de ciclização. ${ }^{53}$

Em relação às benzamidas $\mathbf{1 9}$ e $\mathbf{2 0}$, bem como seus correspondentes produtos de ciclização e hidrogenólise, apesar dos baixos rendimentos de produtos de ciclização, abriu-se uma nova possibilidade de exploração para a obtenção deste tipo de composto, uma vez que, além da possível formação de ciclos de 14 (modo exo) ou 15 membros (modo endo), utilizou-se um radical alquila em vez de arila.

O menor rendimento do produto de ciclização de $\mathbf{1 9}(\mathbf{2 1}, 3 \%)$ em relação ao de $\mathbf{1 3}(\mathbf{1 5}, \mathbf{7 \%})$ pode ser atribuído à maior liberdade conformacional do radical alquila presente em 19. Nesse caso, a cadeia lateral em C-4, na qual é formado o radical, é maior no precursor 19 devido à existência de uma cadeia lateral maior, portanto, 
com maior liberdade conformacional. Assim, poder-se-ia entender que, com a maior liberdade conformacional da cadeia lateral de 19, após a formação do radical alquila, seria entropicamente menos favorável formar o ciclo de 15 membros 21 do que formar o ciclo de 11 membros $\mathbf{1 5}$ a partir de $\mathbf{1 3}$.

Considerando-se que, em relação às 2-iodobenzamidas precursoras 1, 7, 10 e 13, os melhores resultados foram obtidos para a de configuração D-glico 1 , com a cadeia $O$-alílica em C-4 e a cadeia benzamídica em C-6, poder-se-ia esperar que, com a reação radicalar do análogo de 19 com configuração D-glico e com a inversão das posições das cadeias laterais de C-4 e C-6, possam ser obtidos melhores rendimentos de produtos de ciclização.

\section{CONCLUSÃO}

A reação de ciclização radicalar mediada por hidreto de tri- $n$ butilestanho da 2-iodobenzamida alílica 13 forneceu a macrolactama de onze membros $\mathbf{1 5}$ com $7 \%$ de rendimento e o produto de hidrogenólise 33, com 30\% de rendimento. Nas mesmas condições de reação, a 2-iodobenzamida cinamílica 14 forneceu apenas o produto de hidrogenólise $\mathbf{3 4}$, com $30 \%$ de rendimento. A reação de carbociclização radicalar da amida $\mathbf{1 9}$ forneceu a macrolactama de quinze membros $\mathbf{2 1}$, com rendimento de $3 \%$, juntamente com o produto de hidrogenólise 37 , com $26 \%$ de rendimento, enquanto seu análogo cinamílico 20 forneceu apenas o produto de hidrogenólise 38, com $10 \%$ de rendimento. As macrolactamas $\mathbf{1 5}$ e 21 foram formadas pelo modo de ciclização endo, confirmando a tendência geral descrita na literatura.

O baixo rendimento obtido na formação da macrolactama 15 corrobora resultados anteriores do grupo de que a ciclização é mais favorecida quando os grupos $O$-alila e 2 -iodobenzamida se encontram, respectivamente, nas posições 4 e 6 do anel piranosídico. Da mesma forma, a ciclização é mais favorecida quando o carboidrato possui configuração D-glico em vez de D-galacto.

Concluiu-se, por fim, que os precursores $O$-cinamílicos estudados pelo grupo até o presente momento não são adequados para reações de ciclização radicalar, já que não foram obtidos produtos cíclicos das reações de 14 e 20, fato que também ocorreu em trabalhos anteriores.

\section{PARTE EXPERIMENTAL}

\section{Procedimentos gerais}

As temperaturas de fusão foram determinadas em aparelho Microquímica MQAPF 301 e não foram corrigidas. As rotações específicas, $[\alpha]_{\mathrm{D}}$, foram medidas em polarímetros P20 Bellingham + Stanley ou Perkin-Elmer 341 a $25^{\circ} \mathrm{C}$. Todas as substâncias foram identificadas e caracterizadas com base em seus espectros de RMN ${ }^{1} \mathrm{He}{ }^{13} \mathrm{C}$ (complementados com experimentos DEPT, COSY, HMQC e/ou HMBC), registrados em espectrômetros Bruker Avance DPX200 ou DRX400 e utilizando como referência interna o tetrametilsilano. Os espectros no infravermelho foram registrados em aparelho PerkinElmer Spectrum One. As cromatografias em coluna foram realizadas com sílica gel 60, 70-230 mesh (Merck).

\section{6- $O$-Alil-4-azido-2,3-di- $O$-benzil-4-desoxi- $\alpha$-D- galactopiranosídeo de metila (29) e 4-azido-2,3-di- $O$-benzil-6- $O$-cinamil-4-desoxi- $\alpha$-D-galactopiranosídeo de metila (30)}

Em um balão de fundo redondo, foram adicionados $12,5 \mathrm{~mL}$ de solução aquosa de hidróxido de sódio a 50\% (m/v) (0,16 mol), 0,93 g (2,88 mmol) de brometo de tetra- $N$-butilamônio, $40 \mathrm{~mL}$ de diclorometano e 1,0 g (2,50 mmol) de $\mathbf{2 8}$. Em seguida, foram rapidamente gotejados
0,94 mL (1,32 g; 10,9 mmol) de brometo de alila. Fechou-se o balão e manteve-se a mistura sob vigorosa agitação magnética à temperatura ambiente por cerca de $18 \mathrm{~h}$. Após o término da reação, a mistura reagente foi transferida para um funil de separação. Adicionaram-se água $(100 \mathrm{~mL})$ e $50 \mathrm{~mL}$ de clorofórmio. Separaram-se as duas fases e a fase aquosa foi extraída com 2 porções de $100 \mathrm{~mL}$ de clorofórmio. As camadas orgânicas foram reunidas, secadas com sulfato de sódio anidro, filtradas e concentradas até resíduo por destilação sob pressão reduzida. O resíduo obtido foi purificado por cromatografia em coluna de sílica e o produto foi eluído com éter etílico. Foram obtidos 932,8 $\mathrm{mg}(2,12 \mathrm{mmol})$ de $\mathbf{2 9}$, correspondentes a $85 \%$ de rendimento. Óleo amarelado. $[\alpha]_{\mathrm{D}}:+20,8\left(\mathrm{c} 1,25, \mathrm{CHCl}_{3}\right)$. IV $\left(\mathrm{cm}^{-1}\right.$, filme, $\left.\mathrm{v}_{\max }\right): 3065$, 3031, 2913, 2101, 1646, 1605, 1582, 1497, 1454, 1350, 1092. RMN ${ }^{1} \mathrm{H}\left(200 \mathrm{MHz}, \mathrm{CDCl}_{3}\right), \delta:$ 7,37-7,28 (m, $10 \mathrm{H}, \mathrm{H}$ aromáticos), 5,98$5,79(\mathrm{~m}, 1 \mathrm{H}, \mathrm{H}-8), 5,26\left(\mathrm{dd}, 1 \mathrm{H}, J_{\text {trans }}=17,1 \mathrm{~Hz}, J_{\text {geminal }}=1,3 \mathrm{~Hz}, \mathrm{H}-9\right.$ trans $), 5,20\left(\mathrm{dd}, 1 \mathrm{H}, J_{\text {cis }}=10,6 \mathrm{~Hz}, J_{\text {geminal }}=1,3 \mathrm{~Hz}, \mathrm{H}-9 \mathrm{cis}\right), 4,85-4,60$ (m, $4 \mathrm{H}$, H benzílicos), 4,59 (d, $\left.1 \mathrm{H}, J_{1-2}=3,7 \mathrm{~Hz}, \mathrm{H}-1\right), 4,06-3,88$ (m, $5 \mathrm{H}, \mathrm{H}-3, \mathrm{H}-4$, H-5 e H-7), 3,83 (dd, $1 \mathrm{H}, J_{2-3}=9,6 \mathrm{~Hz}, J_{1-2}=3,7 \mathrm{~Hz}$, $\mathrm{H}-2$ ), 3,58-3,45 (m, $2 \mathrm{H}, \mathrm{H}-6$ ), 3,34 (s, $3 \mathrm{H}, \mathrm{H}$ metoxila). RMN ${ }^{13} \mathrm{C}$ (50 MHz, $\mathrm{CDCl}_{3}$ ), $\delta: 138,2,138,0$ (C ipso dos grupos benzila), 134,1 (C-8), 128,32, 128,25, 127,9, 127,7, 127,63 e 127,57 (C aromáticos), 117,3 (C-9), 98,5 (C-1), 77,6 (C-3), 76,0 (C-2), 73,6, 73,0, 72,3 (C-7 e benzílicos), 68,8 (C-6), 67,0 (C-5), 61,3 (C-4), 55,2 (C metoxila).

A reação de 28 com brometo de cinamila, utilizando o mesmo procedimento descrito para a obtenção de $\mathbf{2 9}$, levou a $\mathbf{3 0}$ como um óleo amarelado $(1,23 \mathrm{~g} ; 2,39 \mathrm{mmol} ; 95 \%)$. $[\alpha]_{\mathrm{D}}+9,5\left(c 6,30, \mathrm{CHCl}_{3}\right)$. IV (cm ${ }^{-1}$, filme, $\left.v_{\text {max }}\right)$ : 3062, 3028, 2910, 2101, 1599, 1496, 1453, 1351, 1091. RMN ${ }^{1} \mathrm{H}\left(200 \mathrm{MHz}, \mathrm{CDCl}_{3}\right), \delta: 7,37-7,22(\mathrm{~m}, 15 \mathrm{H}, \mathrm{H}$ aromáticos), 6,59 (d, $\left.1 \mathrm{H}, J_{8-9}=15,9 \mathrm{~Hz}, \mathrm{H}-9\right), 6,25\left(\mathrm{td}, 1 \mathrm{H}, J_{8-9}=\right.$ $\left.15,9 \mathrm{~Hz}, J_{7-8}=6,0 \mathrm{~Hz}, \mathrm{H}-8\right), 4,85-4,79$ ( $\mathrm{m}, 2 \mathrm{H}, \mathrm{H}$ benzílicos), 4,74 (d, $1 \mathrm{H}, J_{\text {geminal }}=11,6 \mathrm{~Hz}, \mathrm{H}$ benzílico $), 4,63\left(\mathrm{~d}, 1 \mathrm{H}, J_{\text {geminal }}=12,0\right.$ $\mathrm{Hz}, \mathrm{H}$ benzílico $), 4,59$ (d, $\left.1 \mathrm{H}, J_{1-2}=3,5 \mathrm{~Hz}, \mathrm{H}-1\right), 4,16\left(\mathrm{~d}, 2 \mathrm{H}, J_{7-8}\right.$ = 6,0 Hz, H-7), 4,07-4,01 (m, 2 H, H-3 e H-4), 3,98-3,91 (m, $1 \mathrm{H}$, $\mathrm{H}-5), 3,84$ (dd, $\left.1 \mathrm{H}, J_{2-3}=9,1 \mathrm{~Hz}, J_{1-2}=3,5 \mathrm{~Hz}, \mathrm{H}-2\right), 3,64-3,50(\mathrm{~m}$, $2 \mathrm{H}, \mathrm{H}-6), 3,34$ (s, $3 \mathrm{H}, \mathrm{H}$ metoxila). RMN ${ }^{13} \mathrm{C}\left(50 \mathrm{MHz}, \mathrm{CDCl}_{3}\right)$, $\delta: 138,2,138,1$ (C ipso dos grupos benzila), 136,5 (C ipso do grupo cinamila), 132,9 (C-9), 128,6, 128,44, 128,37, 128,0, 127,8, 127,74, 127,68 e 126,5 (C aromáticos), 125,4 (C-8), 98,7 (C-1), 77,7 (C-3), 76,1 (C-2), 73,8, 73,1 (C benzílicos), 72,1 (C-7), 68,9 (C-6), 67,1 (C-5), 61,5 (C-4), 55,4 (C metoxila).

\section{6- $O$-Alil-4-amino-2,3-di- $O$-benzil-4-desoxi- $\alpha$-D- galactopiranosídeo de metila (31) e 4-amino-2,3-di- $O$-benzil-6- $O$-cinamil-4-desoxi- $\alpha$-D-galactopiranosídeo de metila (32)}

Em um balão bitubulado de fundo redondo adaptado a um condensador de refluxo, foram colocados $356 \mathrm{mg}$ (9,39 mmol) de hidreto de lítio e alumínio. Sob atmosfera de nitrogênio, resfriamento em banho de gelo e agitação magnética, foram, lentamente, adicionados com seringa 10,5 mL de THF anidro. Em seguida, foi adicionado com seringa $1,0 \mathrm{~g}$ de $\mathbf{2 9}$ (2,28 mmol) dissolvido em $5 \mathrm{~mL}$ de THF anidro. A mistura foi mantida sob agitação magnética por cerca de 2 h. Ao término da reação, foram gotejados $25 \mathrm{~mL}$ de solução aquosa de hidróxido de sódio a $0,4 \mathrm{~mol} / \mathrm{L}$. A mistura reagente foi transferida para um funil de separação, separou-se a fase orgânica e a camada aquosa foi extraída com 5 porções de $20 \mathrm{~mL}$ e 7 porções de $50 \mathrm{~mL}$ de diclorometano. A fase orgânica foi reunida, secada com sulfato de sódio anidro, filtrada e concentrada até a obtenção de $\mathbf{3 1}$ como um resíduo oleoso. Este foi diretamente utilizado na etapa seguinte, sem sofrer nenhum tipo de purificação adicional.

Tratando o azidoéter 30 como descrito para 29, foi obtido $0,46 \mathrm{~g}$ de 32 como um resíduo oleoso, que também foi diretamente utilizado na etapa seguinte sem purificação adicional. 
6- $O$-Alil-2,3-di- $O$-benzil-4-desoxi-4-(2-iodobenzamido)- $\alpha$ D-galactopiranosídeo de metila (13) e 2,3-di- $O$-benzil-6- $O$ cinamil-4-desoxi-4-(2-iodobenzamido)- $\alpha$-D-galactopiranosídeo de metila (14)

Em um balão de fundo redondo adaptado a um condensador de refluxo com tubo dessecador contendo cloreto de cálcio, foram colocados 1,13 g (4,56 mmol) de ácido 2-iodobenzóico e 3,65 mL $(5,95 \mathrm{~g} ; 50,0 \mathrm{mmol})$ de cloreto de tionila. A mistura foi aquecida até refluxo e mantida sob agitação magnética por cerca de $2 \mathrm{~h}$. Ao término da reação, o cloreto de tionila restante foi eliminado por codestilação com benzeno, sob pressão reduzida. O resíduo foi dissolvido em cerca de $10 \mathrm{~mL}$ de diclorometano. Em seguida, em um balão de fundo redondo imerso em banho de gelo, foram adicionados $2 \mathrm{~mL}$ de solução aquosa de hidróxido de sódio a $2 \mathrm{~mol} / \mathrm{L}$ (4,00 mmol) e 0,94 g de 31 bruto dissolvido em cerca de $20 \mathrm{~mL}$ de diclorometano. Sob agitação magnética, gotejou-se a solução de cloreto de 2-iodobenzoíla em diclorometano e, em seguida, mais $8 \mathrm{~mL}$ de solução aquosa de hidróxido de sódio a $2 \mathrm{~mol} / \mathrm{L}(16,0 \mathrm{mmol})$. A mistura foi mantida sob agitação magnética por cerca de $14 \mathrm{~h}$. Ao término da reação, a mistura reagente foi transferida para um funil de separação contendo $25 \mathrm{~mL}$ de água. Separaram-se as camadas aquosa e orgânica e a fase aquosa foi extraída com 3 porções de $25 \mathrm{~mL}$ de diclorometano. As camadas orgânicas foram reunidas, secadas com sulfato de sódio anidro, filtradas e concentradas até resíduo, que foi purificado por cromatografia em coluna de sílica. O produto foi eluído com hexano/ acetato de etila 7:3. Foram obtidos 724,5 $\mathrm{mg}(1,13 \mathrm{mmol})$ de $\mathbf{1 3}$, correspondentes a $49 \%$ de rendimento, e mais $340,7 \mathrm{mg}$ de $\mathbf{1 3} \mathrm{com}$ pequena quantidade de impurezas. Óleo. $[\alpha]_{\mathrm{D}}+37,0\left(c 1,20, \mathrm{CHCl}_{3}\right)$. IV $\left(\mathrm{cm}^{-1}\right.$, filme, $\left.v_{\max }\right): 3299,3062,3030,2908,1649,1584,1508$, $1462,1453,1348,1095 . \mathrm{RMN}^{1} \mathrm{H}\left(200 \mathrm{MHz}, \mathrm{CDCl}_{3}\right), \delta: 7,85$ (d, 1 $\left.\mathrm{H}, J_{\text {orto }}=7,8 \mathrm{~Hz}, \mathrm{H}-13\right), 7,45-7,25(\mathrm{~m}, 12 \mathrm{H}, \mathrm{H}$ aromáticos), 7, 12-7,03 (m, $1 \mathrm{H}, \mathrm{H}-14), 5,97-5,80$ (m, $1 \mathrm{H}, \mathrm{H}-8), 5,92$ (d, $1 \mathrm{H}, J_{\mathrm{NH}-4}=9,1$ $\mathrm{Hz}, \mathrm{H}$ amida), 5,26 (dd, $1 \mathrm{H}, J_{\text {trans }}=17,2 \mathrm{~Hz}, J_{\text {geminal }}=1,4 \mathrm{~Hz}, \mathrm{H}-9$ trans) $, 5,17$ (dd, $1 \mathrm{H}, J_{\text {cis }}=10,6 \mathrm{~Hz}, J_{\text {geminal }}=1,4 \mathrm{~Hz}, \mathrm{H}-9$ cis $), 4,95$ (m, $1 \mathrm{H}, \mathrm{H}-4), 4,94\left(\mathrm{~d}, 1 \mathrm{H}, J_{\text {geminal }}=11,1 \mathrm{~Hz}, \mathrm{H}\right.$ benzílico), 4,85 (d, $1 \mathrm{H}, J_{\text {geminal }}=12,4 \mathrm{~Hz}, \mathrm{H}$ benzílico $), 4,73\left(\mathrm{~d}, 1 \mathrm{H}, J_{\text {geminal }}=12,4 \mathrm{~Hz}\right.$, $\mathrm{H}$ benzílico $), 4,68\left(\mathrm{~d}, 1 \mathrm{H}, J_{1-2}=3,9 \mathrm{~Hz}, \mathrm{H}-1\right), 4,61\left(\mathrm{~d}, 1 \mathrm{H}, J_{\text {geminal }}=\right.$ 11,1 Hz, $\mathrm{H}$ benzílico), 4,21-4,16 (m, $1 \mathrm{H}, \mathrm{H}-5), 4,12-4,07$ (m, $1 \mathrm{H}$, $\mathrm{H}-3), 4,03$ (d, $\left.2 \mathrm{H}, J_{7-8}=5,8 \mathrm{~Hz}, \mathrm{H}-7\right), 3,65\left(\mathrm{~d}, 2 \mathrm{H}, J_{5-6}=5,3 \mathrm{~Hz}\right.$, H-6), 3,53 (dd, $\left.1 \mathrm{H}, J_{2-3}=10,0 \mathrm{~Hz}, J_{1-2}=3,9 \mathrm{~Hz}, \mathrm{H}-2\right), 3,42$ (s, $3 \mathrm{H}$, $\mathrm{H}$ metílicos). $\mathrm{RMN}{ }^{13} \mathrm{C}\left(50 \mathrm{MHz}, \mathrm{CDCl}_{3}\right), \delta: 169,5$ (C-10), 142,2 (C-11), 139,9 (C-13), 138,4 (C ipso dos dois grupos benzila), 134,4 (C-8), 131,1 (C-14), 128,3, 128,2, 128,1, 128,0, 127,7 e 127,5 (C aromáticos), 117,3 (C-9), 98,5 (C-1), 92,1 (C-12), 76,5 (C-3), 74,9 (C-2), 73,3 (C benzílico), 72,4 (C-7), 71,6 (C benzílico), 69,8 (C-6), 67,9 (C-5), 55,4 (C metoxila), 48,7 (C-4).

Pelo tratamento da amina 32 como foi descrito anteriormente para a amina 31, foi obtida a 2-iodobenzamida $\mathbf{1 4}$ como um óleo $(269,6$ $\mathrm{mg}$; 0,37 mmol; 39\%). $[\alpha]_{\mathrm{D}}+25,7\left(c 1,00, \mathrm{CHCl}_{3}\right)$. IV ( $\mathrm{cm}^{-1}$, filme, $\left.v_{\max }\right): 3302,3060,3028,2908,2860,1657,1584,1496,1461,1453$, 1346, 1096. RMN ${ }^{1} \mathrm{H}\left(200 \mathrm{MHz}, \mathrm{CDCl}_{3}\right), \delta: 7,81\left(\mathrm{~d}, 1 \mathrm{H}, J_{\text {orto }}=7,8\right.$ $\mathrm{Hz}, \mathrm{H}-13), 7,45-7,23(\mathrm{~m}, 17 \mathrm{H}, \mathrm{H}$ aromáticos), 7,10-7,00 (m, $1 \mathrm{H}$, H-14), 6,59 (d, $\left.1 \mathrm{H}, J_{8-9}=15,9 \mathrm{~Hz}, \mathrm{H}-9\right), 6,27$ (td, $1 \mathrm{H}, J_{8-9}=15,9$ $\left.\mathrm{Hz}, J_{7-8}=6,1 \mathrm{~Hz}, \mathrm{H}-8\right), 5,91\left(\mathrm{~d}, 1 \mathrm{H}, J_{\mathrm{NH}-4}=10,0 \mathrm{~Hz}, \mathrm{H}\right.$ amida $), 4,94$ (d, $1 \mathrm{H}, J_{\text {geminal }}=11,2 \mathrm{~Hz}, \mathrm{H}$ benzílico), 4,93 (m, $\left.1 \mathrm{H}, \mathrm{H}-4\right), 4,85$ (d, $1 \mathrm{H}, J_{\text {geminal }}=12,1 \mathrm{~Hz}, \mathrm{H}$ benzílico $), 4,72\left(\mathrm{~d}, 1 \mathrm{H}, J_{\text {geminal }}=12,1 \mathrm{~Hz}\right.$, $\mathrm{H}$ benzílico), 4,69 (d, $1 \mathrm{H}, J=3,7 \mathrm{~Hz}, \mathrm{H}-1), 4,61(\mathrm{~d}, 1 \mathrm{H}, J$ = 11,2 Hz, H benzílico), 4,21-4,18 (m, 3 H, H-5 e H-7), 4,09 (dd, $\left.1 \mathrm{H}, J_{3-2}=10,1 \mathrm{~Hz}, J_{3-4}=4,4 \mathrm{~Hz}, \mathrm{H}-3\right), 3,70\left(\mathrm{~d}, 2 \mathrm{H}, J_{5-6}=5,6 \mathrm{~Hz}\right.$, H-6), 3,53 (dd, $\left.1 \mathrm{H}, J_{2-3}=10,1 \mathrm{~Hz}, J_{1-2}=3,7 \mathrm{~Hz}, \mathrm{H}-2\right), 3,43$ (s, $3 \mathrm{H}$, $\mathrm{H}$ metoxila). $\mathrm{RMN}{ }^{13} \mathrm{C}\left(50 \mathrm{MHz}, \mathrm{CDCl}_{3}\right), \delta: 169,5$ (C10), 142,3 (C11), 139,8 (C-13), 138,2 (C ipso dos dois grupos benzila), 136,5 (C ipso do grupo cinamila), 132,9 (C-9), 131,1 (C-14), 128,5, 128,32, 128,25, 128,1, 128,0, 127,8, 127,7, 127,5, 126,5 (C aromáticos), 125,7 (C-8), 98,6 (C-1), 92,2 (C-12), 76,7 (C-3), 75,0 (C-2), 73,3 (C benzílico), 72,2 (C-7), 71,6 (C benzílico), 69,8 (C-6), 68,0 (C-5), 55,4 (C metoxila), 48,7 (C-4).

\section{6-O-Alil-2,3-di- $O$-benzil-4-[3-(cloroacetamido)benzamido]-4- desoxi- $\alpha$-D-galactopiranosídeo de metila (35) e 2,3-di- $O$-benzil- 6- $O$-cinamil-4-[3-(cloroacetamido)benzamido]-4-desoxi- $\alpha$-D- galactopiranosídeo de metila (36)}

Em um balão de fundo redondo adaptado a um condensador de refluxo, foram colocados 5,5 $\mathrm{mL}(5,78 \mathrm{~g}$; $96,3 \mathrm{mmol})$ de ácido acético glacial, $695 \mathrm{mg}(8,47 \mathrm{mmol})$ de acetato de sódio e $0,50 \mathrm{~g}(3,65$ mmol) de ácido 3-aminobenzóico. A mistura, mantida sob agitação mecânica, foi aquecida a $70^{\circ} \mathrm{C}$ até dissolução completa e, em seguida, resfriada até cerca de $45^{\circ} \mathrm{C}$. Foi, então, adicionado $0,34 \mathrm{~mL}(0,48$ g; 4,25 mmol) de cloreto de cloroacetila. A mistura foi resfriada até a temperatura ambiente e foram adicionados $3 \mathrm{~mL}$ de clorofórmio. Após cerca de $3 \mathrm{~h}$, a mistura reagente foi filtrada a vácuo e o sólido retido foi lavado com água e ácido clorídrico diluído. Foram obtidos 744,5 mg (3,49 mmol) de ácido 3-(cloroacetamido)benzóico, na forma de pó branco, correspondentes a $95 \%$ de rendimento. $\mathrm{O}$ sólido obtido foi posteriormente recristalizado em acetato de etila. Em um balão de fundo redondo, foram adicionados $0,85 \mathrm{~mL}(1,39$ g; 11,7 mmol) de cloreto de tionila, $0,2 \mathrm{~mL}$ de clorofórmio e uma gota de piridina. $\mathrm{O}$ balão foi adaptado a um condensador de refluxo e a um tubo dessecador contendo cloreto de cálcio anidro. A mistura foi aquecida e mantida sob agitação magnética entre 55 e $60{ }^{\circ} \mathrm{C}$. Foram, então, adicionados em pequenas porções 489,4 mg (2,29 mmol) de ácido 3-(cloroacetamido)benzóico. Após 3 h, a mistura reagente foi resfriada à temperatura ambiente e adicionaram-se 5 $\mathrm{mL}$ de éter de petróleo (faixa de ebulição: 30-60 ${ }^{\circ} \mathrm{C}$ ). Formou-se um precipitado amarelo que foi filtrado a vácuo e lavado com éter de petróleo. Foram obtidos 223,3 mg (0,96 mmol) de cloreto de 3-(cloroacetamido)benzoíla, correspondentes a $42 \%$ de rendimento. Uma quantidade adicional de 627,1 $\mathrm{mg}$ (2,70 mmol; 91\%) de cloreto de 3-(cloroacetamido)benzoíla foi preparada utilizando-se o mesmo procedimento. Em seguida, toda a quantidade obtida de cloreto de 3-(cloroacetamido)benzoíla (850,4 mg; 3,66 mmol) foi transferida para um balão de fundo redondo e dissolvida em $10 \mathrm{~mL}$ de diclorometano. A mistura reagente foi resfriada em banho de gelo e mantida sob agitação magnética. Foram adicionados $5 \mathrm{~mL}$ de solução aquosa de hidróxido de sódio a 0,92 mol/L (4,60 mmol) e, lentamente, 0,47 $\mathrm{g}(1,14 \mathrm{mmol})$ de 31 que não foi purificado, dissolvido em $13 \mathrm{~mL}$ de diclorometano. Ao término da reação, após cerca de $30 \mathrm{~min}$, a mistura reagente foi refrigerada e, após repouso de $12 \mathrm{~h}$, foi transferida para um funil de separação contendo $25 \mathrm{~mL}$ de água. Separaram-se as duas fases e extraiu-se a fase aquosa com 4 porções de $25 \mathrm{~mL}$ de diclorometano. As fases orgânicas foram reunidas, lavadas com 2 porções de $50 \mathrm{~mL}$ de água destilada, secadas com sulfato de sódio anidro, filtradas e concentradas até resíduo, que foi purificado por cromatografia em coluna de sílica. O produto foi eluído com hexano/ acetato de etila 1:1. Foram obtidos 276,2 $\mathrm{mg}(0,45 \mathrm{mmol})$, de 35 correspondentes a $37 \%$ de rendimento em relação ao precursor azido 29. Óleo amarelado. IV ( $\mathrm{cm}^{-1}$, filme, $\left.v_{\max }\right): 3300,3065,3010,2909$, $1648,1612,1591,1516,1485,1453,1434,1346,1094 . \mathrm{RMN}^{1} \mathrm{H}$ (200 MHz, $\mathrm{CDCl}_{3}$ ), $\delta: ~ 8,62$ (s, $1 \mathrm{H}, \mathrm{H}-\mathrm{N}$ cloroacetamida), 7,86-7,83 (m, 2 H, H-12 e H-14), 7,47-7,21 (m, 12 H, H aromáticos), 6,43 (d, $1 \mathrm{H}, J_{\mathrm{NH}-4}=9,8 \mathrm{~Hz}, \mathrm{H}-\mathrm{N}$ benzamida), 5,92-5,73 (m, $\left.1 \mathrm{H}, \mathrm{H}-8\right), 5,21$ $\left(\mathrm{dd}, 1 \mathrm{H}, J_{\text {geminal }}=1,3 \mathrm{~Hz}, J_{\text {trans }}=17,3 \mathrm{~Hz}, \mathrm{H}-9\right.$ trans $), 5,12(\mathrm{dd}, 1 \mathrm{H}$, $\left.J_{\text {cis }}=10,3 \mathrm{~Hz}, J_{\text {geminal }}=1,3 \mathrm{~Hz}, \mathrm{H}-9 \mathrm{cis}\right), 4,97\left(\mathrm{dd}, 1 \mathrm{H}, J_{\mathrm{NH}-4}=9,8\right.$ $\left.\mathrm{Hz}, J_{3-4}=3,9 \mathrm{~Hz}, \mathrm{H}-4\right), 4,88\left(\mathrm{~d}, 1 \mathrm{H}, J_{\text {geminal }}=11,3 \mathrm{~Hz}, \mathrm{H}\right.$ benzílico), 
4,85 (d, $1 \mathrm{H}, J_{\text {geminal }}=12,0 \mathrm{~Hz}, \mathrm{H}$ benzílico), 4,74 (d, $1 \mathrm{H}, J_{1-2}=3,6$ $\mathrm{Hz}, \mathrm{H}-1), 4,67$ (d, $1 \mathrm{H}, J_{\text {geminal }}=12,0 \mathrm{~Hz}, \mathrm{H}$ benzílico), 4,59 (d, $1 \mathrm{H}$, $J_{\text {geminal }}=11,3 \mathrm{~Hz}$, H benzílico), 4,20-4,04 (m, 4 H, H-3, H-5 e H-18), $3,95\left(\mathrm{dd}, 2 \mathrm{H}, J_{7-8}=5,4 \mathrm{~Hz}, J_{7-9}=1,0 \mathrm{~Hz}, \mathrm{H}-7\right), 3,63\left(\mathrm{dd}, 1 \mathrm{H}, J_{2-3}=\right.$ $\left.10,0 \mathrm{~Hz}, J_{1-2}=3,6 \mathrm{~Hz}, \mathrm{H}-2\right), 3,57-3,53(\mathrm{~m}, 2 \mathrm{H}, \mathrm{H}-6), 3,42(\mathrm{~s}, 3 \mathrm{H}, \mathrm{H}$ metoxila). $\mathrm{RMN}{ }^{13} \mathrm{C}\left(50 \mathrm{MHz}, \mathrm{CDCl}_{3}\right), \delta: 167,4,164,2$ (C-10, C-17), 138,1, 138,0 (C ipso dos grupos benzila), 137,3 (C-13), 135,4 (C-11), 134,2 (C-8), 129,3 (C-15), 128,2, 128,1, 127,9, 127,8, 127,6, 127,4 (C aromáticos), 123,0, 122,9 (C-14 e C-16), 118,9 (C-12), 117,2 (C-9), 98,6 (C-1), 76,4 (C-3), 75,4 (C-2), 73,4 (C benzílico), 72,3 (C-7), 71,5 (C benzílico), 69,5 (C-6), 67,7 (C-5), 55,3 (C metoxila), 48,8 (C-4), 42,8 (C-18).

Após o tratamento da amina 32 seguindo o mesmo procedimento descrito para $\mathbf{3 1}$, foi obtida a cloroacetamida $\mathbf{3 6}$ como um óleo amarelado (227,2 mg; 0,33 mmol; 41\% em relação a 30). IV ( $\mathrm{cm}^{-1}$, filme, $\left.v_{\max }\right): 3312,3062,3029,2924,2860,1691,1649,1612,1591,1547$, $1519,1485,1453,1434,1347,1096 . \mathrm{RMN}^{1} \mathrm{H}\left(200 \mathrm{MHz}, \mathrm{CDCl}_{3}\right)$, $\delta$ : 8,51 (s, $1 \mathrm{H}, \mathrm{H}-\mathrm{N}$ cloroacetamida), 7,83-7,79 (m, $2 \mathrm{H}, \mathrm{H}-12$ e H-14), 7,47-7,20 (m, 17 H, H aromáticos), 6,55-6,47 (m, 2 H, H-9 e H-N benzamida), 6,17 (td, $1 \mathrm{H}, J_{8-9}=15,9 \mathrm{~Hz}, J_{7-8}=6,1 \mathrm{~Hz}, \mathrm{H}-8$ ), $5,00\left(\mathrm{dd}, 1 \mathrm{H}, J_{\mathrm{NH}-4}=9,4 \mathrm{~Hz}, J_{3-4}=3,6 \mathrm{~Hz}, \mathrm{H}-4\right), 4,92-4,81(\mathrm{~m}, 2 \mathrm{H}$, $\mathrm{H}$ benzílicos), 4,74 (d, $\left.1 \mathrm{H}, J_{1-2}=3,6 \mathrm{~Hz}, \mathrm{H}-1\right), 4,66$ (d, $1 \mathrm{H}, J_{\text {geminal }}=$ 12,2 Hz, H benzílico), 4,59 (d, $1 \mathrm{H}, J_{\text {geminal }}=11,2 \mathrm{~Hz}, \mathrm{H}$ benźlico), 4,22-4,04 (m, 6 H, H-3, H-5, H-7 e H-18), 3,67-3,53 (m, 3 H, H-2 e H-6), 3,42 (s, $3 \mathrm{H}, \mathrm{H}$ metoxila). $\mathrm{RMN}{ }^{13} \mathrm{C}\left(50 \mathrm{MHz}, \mathrm{CDCl}_{3}\right), \delta$ : 167,5, 164,2 (C-10 e C-17), 138,2, 138,1 (C ipso dos grupos benzila), 137,2 (C-13), 136,3 (C ipso do grupo cinamila), 135,4 (C-11), 132,7 (C-9), 129,3 (C-15), 128,4, 128,23, 128,17, 127,92, 127,88, 127,65, 127,57, 127,4 e 126,3 (C aromáticos), 125,4 (C-8), 123,1, 123,0 (C14 e C-16), 118,9 (C-12), 98,7 (C-1), 76,4 (C-3), 75,4 (C-2), 73,5 (C benzílico), 72,0 (C-7), 71,5 (C benzílico), 69,4 (C-6), 67,7 (C-5), 55,4 (C metoxila), 48,9 (C-4), 42,8 (C-18).

\section{6- $O$-Alil-2,3-di- $O$-benzil-4-desoxi-4-[3-(iodoacetamido) benzamido]- $\alpha$-D-galactopiranosídeo de metila (19) e 2,3-di- $O$ - Benzil-6- $O$-cinamil-4-desoxi-4-[3-(iodoacetamido)benzamido]- $\alpha$-D-galactopiranosídeo de metila (20)}

Em um balão de fundo redondo foram colocados $293,1 \mathrm{mg}(0,48$ $\mathrm{mmol})$ de $35,2 \mathrm{~mL}$ de acetona, $564 \mathrm{mg}(3,76 \mathrm{mmol})$ de iodeto de sódio e, posteriormente, mais $1 \mathrm{~mL}$ de acetona. A mistura foi mantida sob agitação mecânica à temperatura ambiente por cerca de $24 \mathrm{~h}$. A mistura reagente foi, então, transferida para cápsula de porcelana e concentrada até resíduo, sob corrente de ar quente. Em seguida, o resíduo foi retomado com $30 \mathrm{~mL}$ de diclorometano, transferido para funil de separação e lavado com 2 porções de $15 \mathrm{~mL}$ de água destilada. A camada orgânica foi lavada com $20 \mathrm{~mL}$ de água, secada com sulfato de sódio anidro, filtrada e concentrada até resíduo. Foram obtidos $311,3 \mathrm{mg}(0,44 \mathrm{mmol})$ de $\mathbf{1 9}$, correspondentes a $92 \%$ de rendimento. Sólido amarelado. PF: $46,3-48,6^{\circ} \mathrm{C}$. $[\alpha]_{\mathrm{D}}+28,9\left(c 1,10, \mathrm{CHCl}_{3}\right)$. IV $\left(\mathrm{cm}^{-1}\right.$, filme, $\left.v_{\text {max }}\right): 3292,3062,2908,1645,1612,1590,1553,1519$, $1484,1453,1432,1346,1093 . \mathrm{RMN}^{1} \mathrm{H}\left(200 \mathrm{MHz}, \mathrm{CDCl}_{3}\right), \delta: 9,28$ (s, $1 \mathrm{H}, \mathrm{H}-\mathrm{N}$ iodoacetamida), 7,95 (s, $1 \mathrm{H}, \mathrm{H}-12), 7,87$ (d, $1 \mathrm{H}, J_{\text {orto }}$ = 7,6 Hz, H-14), 7,33-7,17 (m, $12 \mathrm{H}, \mathrm{H}$ aromáticos $), 6,56(\mathrm{~d}, 1 \mathrm{H}$, $J_{\mathrm{NH}-4}=9,8 \mathrm{~Hz}, \mathrm{H}-\mathrm{N}$ benzamida), 5,91-5,71 (m, $\left.1 \mathrm{H}, \mathrm{H}-8\right), 5,20$ (dd, $1 \mathrm{H}, J_{\text {trans }}=17,3 \mathrm{~Hz}, J_{\text {geminal }}=1,1 \mathrm{~Hz}, \mathrm{H}-9$ trans $), 5,11\left(\mathrm{dd}, 1 \mathrm{H}, J_{\text {cis }}=\right.$ $\left.10,6 \mathrm{~Hz}, J_{\text {geminal }}=1,1 \mathrm{~Hz}, \mathrm{H}-9 \mathrm{cis}\right), 4,99\left(\mathrm{dd}, 1 \mathrm{H}, J_{\mathrm{NH}-4}=9,8 \mathrm{~Hz}, J_{3-4}\right.$ $=4,1 \mathrm{~Hz}, \mathrm{H}-4), 4,88\left(\mathrm{~d}, 1 \mathrm{H}, J_{\text {geminal }}=11,2 \mathrm{~Hz}, \mathrm{H}\right.$ benzílico $), 4,85(\mathrm{~d}$, $1 \mathrm{H}, J_{\text {geminal }}=11,8 \mathrm{~Hz}, \mathrm{H}$ benzílico $), 4,75\left(\mathrm{~d}, 1 \mathrm{H}, J_{1-2}=3,5 \mathrm{~Hz}, \mathrm{H}-1\right)$, 4,70-4,59 (m, $2 \mathrm{H}, \mathrm{H}$ benzílicos), 4,19 (t, $\left.1 \mathrm{H}, J_{5-6}=5,6 \mathrm{~Hz}, \mathrm{H}-5\right)$, 4,09 (dd, $\left.1 \mathrm{H}, J_{2-3}=10,1 \mathrm{~Hz}, J_{3-4}=4,1 \mathrm{~Hz}, \mathrm{H}-3\right), 3,96-3,94(\mathrm{~m}, 2 \mathrm{H}$, H-7), 3,82 (s, $2 \mathrm{H}, \mathrm{H}-18), 3,68\left(\mathrm{dd}, 1 \mathrm{H}, J_{2-3}=10,1 \mathrm{~Hz}, J_{1-2}=3,5 \mathrm{~Hz}\right.$, $\mathrm{H}-2), 3,57-3,53$ (m, $2 \mathrm{H}, \mathrm{H}-6), 3,41$ (s, $3 \mathrm{H}, \mathrm{H}$ metoxila). RMN ${ }^{13} \mathrm{C}$
(50 MHz, $\mathrm{CDCl}_{3}$ ), $\delta: 168,2,166,7$ (C-10 e C-17), 138,6 (C-13), 138,2, 138,1 (C ipso dos grupos benzila), 135,2 (C-11), 134,2 (C-8), 129,3 (C-15), 128,4, 128,3, 128,0, 127,8, 127,5 (C aromáticos), 123,3, 122,4 (C-14 e C-16), 119,1 (C-12), 117,5 (C-9), 98, 7 (C-1), 76,5 (C-3), 75, 7 (C-2), 73,6 (C benzílico), 72,4 (C-7), 71,8 (C benzílico), 69,4 (C-6), 67,7 (C-5), 55,5 (C metoxila), 49,2 (C-4), 0,4 (C-18).

Este mesmo procedimento foi usado para obtenção da iodoacetamida 20, um sólido esbranquiçado, a partir da cloroacetamida $\mathbf{3 6}$ (139,0 mg; 0,18 mmol; 57\%). PF: 66,2-72,0 ${ }^{\circ} \mathrm{C}$. $[\alpha]_{\mathrm{D}}+28,6(c$ 1,00, $\left.\mathrm{CHCl}_{3}\right)$. IV ( $\mathrm{cm}^{-1}$, filme, $\left.v_{\text {max }}\right): 3296,3028,2907,1645,1612,1590$, 1554, 1516, 1485, 1452, 1431, 1347, 1094. RMN ${ }^{1} \mathrm{H}(200 \mathrm{MHz}$, $\mathrm{CDCl}_{3}$ ), $\delta: 8,89$ (s, $1 \mathrm{H}, \mathrm{H}-\mathrm{N}$ iodoacetamida), 7,90-7,83 (m, $2 \mathrm{H}, \mathrm{H}-12$ e H-14), 7,34-7,18 (m, 17 H, H aromáticos), 6,56-6,48 (m, 2 H, H-N benzamida e H-9), 6,16 (td, $\left.1 \mathrm{H}, J_{8-9}=15,8 \mathrm{~Hz}, J_{7-8}=6,0 \mathrm{~Hz}, \mathrm{H}-8\right)$, $5,02\left(\mathrm{dd}, 1 \mathrm{H}, J_{\mathrm{NH}-4}=9,3 \mathrm{~Hz}, J_{3-4}=3,1 \mathrm{~Hz}, \mathrm{H}-4\right), 4,89$ (d, $1 \mathrm{H}, J_{\text {geminal }}$ $=11,0 \mathrm{~Hz}, \mathrm{H}$ benzílico), 4,84 (d, $1 \mathrm{H}, J_{\text {geminal }}=11,8 \mathrm{~Hz}, \mathrm{H}$ benzílico), 4,74 (d, $1 \mathrm{H}, J_{1-2}=3,6 \mathrm{~Hz}, \mathrm{H}-1$ ), 4,69-4,59 (m, $2 \mathrm{H}, \mathrm{H}$ benzílicos), 4,24-4,05 (m, 4 H, H-3, H-5 e H-7), 3,79 (s, 2 H, H-18), 3,67-3,53 (m, $3 \mathrm{H}, \mathrm{H}-2$ e H-6), 3,42 (s, $3 \mathrm{H}, \mathrm{H}$ metoxila). RMN ${ }^{13} \mathrm{C}(50 \mathrm{MHz}$, $\mathrm{CDCl}_{3}$ ), $\delta: 168,0,166,2$ (C-10 e C-17), 138,4, 138,2, 138,1 (C ipso dos grupos benzila e C-13), 136,4 (C ipso do grupo cinamila), 135,1 (C-11), 132,9 (C-9), 129,0 (C-15), 128,5, 128,3, 128,2, 128,0, 127,79, 127,75, 127,4, 126,4 (C aromáticos), 125,4 (C-8), 123,4, 122,6 (C14 e C-16), 119,1 (C-12), 98,7 (C-1), 76,4 (C-3), 75,6 (C-2), 73,6 (C benzílico), 72,1 (C-7), 71,8 (C benzílico), 69,3 (C-6), 67,6 (C-5), 55,5 (C metílico), 49,1 (C-4), 0,0 (C-18).

Reação radicalar com 6-O-alil-2,3-di- $O$-benzil-4-desoxi-4(2-iodobenzamido)- $\alpha$-D-galactopiranosídeo de metila (13), 2,3-di- $O$-benzil-6- $O$-cinamil-4-desoxi-4-(2-iodobenzamido)$\alpha$-D-galactopiranosídeo de metila (14), 6-O-alil-2,3-di$O$-benzil-4-desoxi-4-[3-(iodoacetamido)benzamido]- $\alpha-D-$ galactopiranosídeo de metila (19) e 2,3-di- $O$-benzil-6- $O$ cinamil-4-desoxi-4-[3-(iodoacetamido)benzamido]- $\alpha-D-$ galactopiranosídeo de metila (20)

Um balão de fundo redondo tritubulado foi adaptado, por duas de suas bocas, a um funil de adição e a um condensador de refluxo, sendo este último, por sua vez, adaptado a um tubo para escape de gás. A outra boca do balão e a do funil de adição foram fechadas com rolhas de borracha e o sistema foi colocado sob atmosfera de nitrogênio. Em seguida, adicionou-se ao balão, com auxílio de seringa, uma solução de 13 (204 mg; 0,32 mmol) dissolvido em $45 \mathrm{~mL}$ de benzeno anidro. O sistema foi aquecido até refluxo, à temperatura entre 90 e $95^{\circ} \mathrm{C}$, em banho de óleo e sob agitação magnética. Com auxílio de seringa, foi injetada no funil de adição uma solução de AIBN (22,0 mg; 0,13 mmol) e $\mathrm{Bu}_{3} \mathrm{SnH}(0,128 \mathrm{~mL} ; 138 \mathrm{mg} ; 0,47 \mathrm{mmol})$ dissolvidos em $15 \mathrm{~mL}$ de benzeno anidro. O conteúdo do funil de adição foi lenta e cuidadosamente gotejado durante cerca de $5 \mathrm{~h}$ à solução contida no balão. Ao término do gotejamento, a mistura reagente foi mantida sob aquecimento e atmosfera de nitrogênio por mais $1 \mathrm{~h}$ e, em seguida, foi transferida para um balão de fundo redondo. O solvente foi eliminado sob pressão reduzida e o resíduo foi submetido a várias purificações por cromatografia em coluna de sílica. Foram isolados os produtos de ciclização 11-endo 15 (12,1 mg; 23 mmol; 7\%) e de hidrogenólise 33 (49,8 mg; 96 mmol; 30\%). Macrolactama (15). Sólido branco. PF: $54,7-56,6^{\circ} \mathrm{C} .[\alpha]_{\mathrm{D}}+96,1\left(c 1,00, \mathrm{CHCl}_{3}\right)$. IV $\left(\mathrm{cm}^{-1}\right.$, filme, $\left.v_{\max }\right)$ : 3301, 3063, 3030, 2917, 2865, 1645, 1600, 1497, 1453, 1094. RMN ${ }^{1} \mathrm{H}\left(400 \mathrm{MHz}_{\mathrm{CDCl}}\right.$ ), $\delta:$ 7,47-7,15 (m, $14 \mathrm{H}, \mathrm{H}$ aromáticos), 6,03 $\left(\mathrm{d}, 1 \mathrm{H}, J_{\mathrm{NH}-4}=10,4 \mathrm{~Hz}, \mathrm{H}-\mathrm{N}\right), 5,07$ (ddd, $1 \mathrm{H}, J_{\mathrm{NH}-4}=10,4 \mathrm{~Hz}, J_{3-4}$ $\left.=4,6 \mathrm{~Hz}, J_{4-5}=1,4 \mathrm{~Hz}, \mathrm{H}-4\right), 4,91-4,82$ ( $\mathrm{m}, 2 \mathrm{H}, \mathrm{H}$ benzílicos), 4,70 $\left(\mathrm{d}, 1 \mathrm{H}, J_{\text {geminal }}=12,0 \mathrm{~Hz}, \mathrm{H}\right.$ benzílico $), 4,66\left(\mathrm{~d}, 1 \mathrm{H}, J_{1-2}=3,9 \mathrm{~Hz}\right.$, $\mathrm{H}-1), 4,62$ (d, $1 \mathrm{H}, J_{\text {geminal }}=11,2 \mathrm{~Hz}, \mathrm{H}$ benzílico), 4,03 (dd, $1 \mathrm{H}, J_{2-3}$ 
$\left.=10,2 \mathrm{~Hz}, J_{3-4}=4,6 \mathrm{~Hz}, \mathrm{H}-3\right), 3,90-3,89$ (m, $1 \mathrm{H}, \mathrm{H}-5$ ), 3,80 (dd, 1 $\left.\mathrm{H}, J_{\text {geminal }}=10,8 \mathrm{~Hz}, J_{5-6}=3,6 \mathrm{~Hz}, \mathrm{H}-6\right), 3,73\left(\mathrm{dd}, 1 \mathrm{H}, J_{\text {geminal }}=10,8\right.$ $\mathrm{Hz}, J_{5-6}=1,2 \mathrm{~Hz}, \mathrm{H}-6$ ') , 3,65-3,61 (m, $\left.1 \mathrm{H}, \mathrm{H}-7\right), 3,53$ (dd, $1 \mathrm{H}, J_{2-3}$ $\left.=10,2 \mathrm{~Hz}, J_{1-2}=3,9 \mathrm{~Hz}, \mathrm{H}-2\right), 3,36(\mathrm{~s}, 3 \mathrm{H}, \mathrm{H}$ metoxila), 3,29-3,23 (m, $\left.1 \mathrm{H}, \mathrm{H}-7^{\prime}\right), 3,12$ (ddd, $1 \mathrm{H}, J_{\text {geminal }}=14,1 \mathrm{~Hz}, J_{8-9}=7,3 \mathrm{~Hz}, J_{8^{\prime}-9}$ = 3,1 Hz, H-9), 2,95-2,89 (m, 1 H, H-9'), 2,07-1,98 (m, 1 H, H-8), 1,71-1,63 (m, $\left.1 \mathrm{H}, \mathrm{H}-8^{\prime}\right)$. RMN ${ }^{13} \mathrm{C}\left(100 \mathrm{MHz}, \mathrm{CDCl}_{3}\right), \delta: 172,5$ (C10), 141,1 (C-12), 138,4, 138,3 (C ipso dos grupos benzila), 137,2 (C-11), 130,9, 129,9, 128,6, 128,5, 128,4, 128,24, 128,20, 128,1, $127,9,127,8,127,6,126,8,125,8$ (C aromáticos), 99,2 (C-1), 75,8 (C-3), 75,3 (C-2), 73,7 (C benzílico), 72,6 (C-7), 72,2 (C-6), 71,2 (C benzílico), 66,7 (C-5), 55,6 (C metoxila), 49,7 (C-4), 30,8 (C-8), 29,9 (C-9). Produto de hidrogenólise (33). Óleo. $[\alpha]_{\mathrm{D}}+65,3$ ( c 0,76, $\mathrm{CHCl}_{3}$ ). IV ( $\mathrm{cm}^{-1}$, filme, $\left.v_{\text {max }}\right): 3326,3062,3030,2913,2865,1646$, $1602,1580,1516,1453,1095$. RMN ${ }^{1} \mathrm{H}\left(200 \mathrm{MHz}, \mathrm{CDCl}_{3}\right), \delta: 7,75-$ 7,71 (m, 2 H, H-12), 7,56-7,21 (m, 14 H, H aromáticos), 6,22 (d, 1 H, $\left.J_{\mathrm{NH}-4}=10,3 \mathrm{~Hz}, \mathrm{H}-\mathrm{N}\right), 5,93-5,74(\mathrm{~m}, 1 \mathrm{H}, \mathrm{H}-8), 5,22$ (dd, $1 \mathrm{H}, J_{\text {trans }}$ $=17,2 \mathrm{~Hz}, J_{\text {geminal }}=1,6 \mathrm{~Hz}, \mathrm{H}-9$ trans $), 5,13\left(\mathrm{dd}, 1 \mathrm{H}, J_{\text {cis }}=10,4 \mathrm{~Hz}\right.$, $J_{\text {geminal }}=1,6 \mathrm{~Hz}, \mathrm{H}-9$ cis $), 5,00-4,95(\mathrm{~m}, 1 \mathrm{H}, \mathrm{H}-4), 4,89\left(\mathrm{~d}, 1 \mathrm{H}, J_{\text {geminal }}\right.$ $=11,3 \mathrm{~Hz}, \mathrm{H}$ benzílico), 4,86 (d, $1 \mathrm{H}, J_{\text {geminal }}=12,1 \mathrm{~Hz}, \mathrm{H}$ benzílico), 4,73-4,72 (m, $1 \mathrm{H}, \mathrm{H}-1), 4,69$ (d, $1 \mathrm{H}, J_{\text {geminal }}^{\text {geminal }}=12,1 \mathrm{~Hz}, \mathrm{H}$ benzílico), $4,58\left(\mathrm{~d}, 1 \mathrm{H}, J_{\text {geminal }}=11,3 \mathrm{~Hz}, \mathrm{H}\right.$ benzílico $), 4,20-4,15(\mathrm{~m}, 1 \mathrm{H}, \mathrm{H}-5)$, $4,07\left(\mathrm{dd}, 1 \mathrm{H}, J_{2-3}=10,0 \mathrm{~Hz}, J_{3-4}=4,4 \mathrm{~Hz}, \mathrm{H}-3\right), 3,98-3,95(\mathrm{~m}, 2$ H, H-7), 3,63-3,50 (m, 3 H, H-2 e H-6), 3,43 (s, 3 H, H metoxila). $\mathrm{RMN}{ }^{13} \mathrm{C}\left(50 \mathrm{MHz}, \mathrm{CDCl}_{3}\right), \delta: 167,9$ (C-10), 138,3, 138,2 (C ipso dos grupos benzila), 134,3 (C-11), 131,6 (C-8), 128,6, 128,4, 128,3, 128,1, 127,8, 127,5, 127,0 (C aromáticos), 117,3 (C-9), 98,7 (C-1), 76,5, 75,3 (C-2 e C-3), 73,6, 72,4, 71,5 (C-7 e C benzílicos), 69,7 (C-6), 68,0 (C-5), 55,4 (C metoxila), 48,7 (C-4).

Por tratamento da 2-iodobenzamida $\mathbf{1 4}$ como foi descrito para 13, foi obtido o produto de hidrogenólise 34 (98,1 mg; $0,17 \mathrm{mmol}$; $30 \%$ ), como um sólido. $[\alpha]_{\mathrm{D}}+39,6\left(c 1,17, \mathrm{CHCl}_{3}\right)$. IV ( $\mathrm{cm}^{-1}$, filme, $\left.v_{\max }\right): 3316,3061,3029,2916,2865,1655,1601,1580,1514,1452$, 1097. RMN ${ }^{1} \mathrm{H}\left(400 \mathrm{MHz}, \mathrm{CDCl}_{3}\right), \delta: 7,72\left(\mathrm{~d}, 2 \mathrm{H}, J_{\text {orto }}=7,2 \mathrm{~Hz}\right.$, $\mathrm{H}-12), 7,50-7,06$ (m, $18 \mathrm{H}, \mathrm{H}$ aromáticos), 6,53 (d, $1 \mathrm{H}, J_{8-9}=16,4$ Hz, H-9), 6,29-6, 17 (m, 2 H, H-N e H-8), 5,00-4,95 (m, 1 H, H-4), 4,91-4,84 (m, 2 H, H benzílicos), 4,73-4,67 (m, 2 H, H-1 e H benzílico 2- $O$-benzil), 4,58 (d, $1 \mathrm{H}, J_{\text {geminal }}=11,6 \mathrm{~Hz}, \mathrm{H}$ benzílico 3- $O$-benzil), 4,19-4,07 (m, 4 H, H-3, H-5 e H-7), 3,70-3,63 (m, 2 H, H-6), 3,573,54 (m, $1 \mathrm{H}, \mathrm{H}-2), 3,43$ (s, $3 \mathrm{H}, \mathrm{H}$ metoxila). RMN ${ }^{13} \mathrm{C}(100 \mathrm{MHz}$, $\mathrm{CDCl}_{3}$ ), $\delta: 168,0$ (C-10), 138,3, 138,2 (C ipso dos grupos benzila), 136,6 (C ipso do grupo cinamila), 134,7 (C-11), 132,9 (C-9), 131,6 (C-14), 128,6, 128,5, 128,4, 128,2, 128,1, 127,8, 127,7, 127,5 (C aromáticos, C-13 e C-13'), 127,0 (C-12 e C-12'), 126,5 (C orto do grupo cinamila), 125,7 (C-8), 98,7 (C-1), 76,5 (C-3), 75,4 (C-2), 73,6 (C benzílico 2- $O$-benzil), 72,2 (C-7), 71,6 (C benzílico 3- $O$-benzil), 69,7 (C-6), 68,0 (C-5), 55,5 (C metoxila), 48,8 (C-4).

$\mathrm{O}$ mesmo procedimento descrito para a reação de ciclizaçao radicalar de $\mathbf{1 3}$ foi aplicado à iodoacetamida 19. Foram obtidos a macrolactama formada por ciclização 15-endo 21 (1,7 mg; 3,0 mmol; 3\%) e o produto de hidrogenólise 37 (14,7 mg; $26 \mathrm{mmol}$; 26\%). Macrolactama 21. Sólido branco. PF: $222,3-224,1{ }^{\circ} \mathrm{C}$. $[\alpha]$ D $-33,0\left(c 0,28, \mathrm{CHCl}_{3}\right)$. IV ( $\mathrm{cm}^{-1}$, filme, $\left.v_{\max }\right)$ : 3298, 3063, 3022, 2913, 2865, 1643, 1609, 1454, 1095. RMN ${ }^{1} \mathrm{H}\left(400 \mathrm{MHz}, \mathrm{CDCl}_{3}\right)$, $\delta: 10,14\left(\mathrm{~s}, 1 \mathrm{H}, \mathrm{H}-\mathrm{N}\right.$ pentanamida), $8,67\left(\mathrm{dd}, 1 \mathrm{H}, J_{\text {orto }}=8,1 \mathrm{~Hz}\right.$, $\left.J_{\text {meta }}=1,0 \mathrm{~Hz}, \mathrm{H}-14\right), 8,26(\mathrm{~s}, 1 \mathrm{H}, \mathrm{H}-12), 7,41\left(\mathrm{t}, 1 \mathrm{H}, J_{\text {orto }}=8,0\right.$ $\mathrm{Hz}, \mathrm{H}-15), 7,32-7,10(\mathrm{~m}, 11 \mathrm{H}, \mathrm{H}$ aromáticos $), 6,46\left(\mathrm{~d}, 1 \mathrm{H}, J_{\mathrm{NH}-4}=\right.$ $10,2 \mathrm{~Hz}, \mathrm{H}-\mathrm{N}$ benzamida), 5,04 (dd, $1 \mathrm{H}, J_{\mathrm{NH}-4}=10,2 \mathrm{~Hz}, J_{3-4}=3,7$ $\mathrm{Hz}, \mathrm{H}-4), 4,87$ (d, $1 \mathrm{H}, J_{\text {geminal }}=12,0 \mathrm{~Hz}, \mathrm{H}$ benzílico 3-O-benzil), $4,82\left(\mathrm{~d}, 1 \mathrm{H}, J_{\text {geminal }}=11,8 \mathrm{~Hz}, \mathrm{H}\right.$ benzílico 2-O-benzil), 4,68-4,64 ( $\mathrm{m}, 3 \mathrm{H}, \mathrm{H}-1, \mathrm{H}$ benzílico 2-O-benzil e $\mathrm{H}$ benzílico 3-O-benzil), $4,16\left(\mathrm{dd}, 1 \mathrm{H}, J_{5-6}=9,6 \mathrm{~Hz}, J_{5-6}=4,8 \mathrm{~Hz}, \mathrm{H}-5\right), 4,01$ (dd, $1 \mathrm{H}, J_{2-3}$ $\left.=10,2 \mathrm{~Hz}, J_{3-4}=3,7 \mathrm{~Hz}, \mathrm{H}-3\right), 3,61-3,56(\mathrm{~m}, 2 \mathrm{H}, \mathrm{H}-2$ e H-7), 3,51-
3,43 (m, 2 H, H-6 e H-6'), 3,41 (s, 3 H, H metoxila), 3,34-3,29 (m, $1 \mathrm{H}, \mathrm{H}-7$ ') , 2,75-2,67 (m, $1 \mathrm{H}, \mathrm{H}-18$ ), 2,33-2,26 (m, $1 \mathrm{H}, \mathrm{H}-18$ '), 2,03-1,97 (m, 1 H, H-9), 1,72-1,66 (m, 3 H, H-8 e H-9'). RMN ${ }^{13} \mathrm{C}$ $\left(100 \mathrm{MHz}, \mathrm{CDCl}_{3}\right), \delta: 173,3$ (C-17), 168,3 (C-10), 140,9 (C-13), 138,3, 137,9 (C ipso dos grupos benzila), 134,1 (C-11), 129,3 (C15), 128,4, 128,3, 128,2, 128,0, 127,8, 127,6 (C aromáticos), 123,4 (C-14), 119,9 (C-12), 119,4 (C-16), 99,2 (C-1), 76,14 (C-2), 76,09 (C-3), 73,8 (C benzílico 3-O-benzil), 72,3 (C-7), 72,0 (C benzílico 2-O-benzil), 68,2 (C-6), 66,5 (C-5), 55,7 (C metoxila), 48,4 (C4), 38,0 (C-18), 28,9 (C-8), 25,0 (C-9). Produto de hidrogenólise (37). Sólido branco. PF: 86,7-89,2 ${ }^{\circ} \mathrm{C}$. $[\alpha]_{\mathrm{D}}+54,7\left(c 1,50, \mathrm{CHCl}_{3}\right)$. IV $\left(\mathrm{cm}^{-1}\right.$, filme, $\left.v_{\text {max }}\right): 3305,3064,3027,2916,2865,1645,1610$, 1497, 1453, 1094. RMN ${ }^{1} \mathrm{H}\left(400 \mathrm{MHz}, \mathrm{CDCl}_{3}\right), \delta: 7,87$ (d, $1 \mathrm{H}$, $J_{\text {orto }}=7,2 \mathrm{~Hz}, \mathrm{H}-14$ ou H-16), 7,73 (s, $\left.1 \mathrm{H}, \mathrm{H}-12\right), 7,42-7,21$ (m, $13 \mathrm{H}, \mathrm{H}$ aromáticos e $\mathrm{H}-\mathrm{N}$ acetamida), $6,30\left(\mathrm{~d}, 1 \mathrm{H}, J_{\mathrm{NH}-4}=9,7 \mathrm{~Hz}\right.$, $\mathrm{H}-\mathrm{N}$ benzamida), 5,88-5,78 (m, $1 \mathrm{H}, \mathrm{H}-8), 5,22\left(\mathrm{dd}, 1 \mathrm{H}, J_{\text {trans }}=\right.$ $17,2 \mathrm{~Hz}, J_{\text {geminal }}=1,6 \mathrm{~Hz}, \mathrm{H}-9$ trans $), 5,12\left(\mathrm{dd}, 1 \mathrm{H}, J_{\text {cis }}=10,4 \mathrm{~Hz}\right.$, $\left.J_{\text {geminal }}=1,6 \mathrm{~Hz}, \mathrm{H}-9 \mathrm{cis}\right), 4,94\left(\mathrm{dd}, 1 \mathrm{H}, J_{\mathrm{NH}-4}=9,7 \mathrm{~Hz}, J_{3-4}=4,7\right.$ Hz, H-4), 4,89-4,83 (m, 2 H, H benzílico 2-O-benzil e H benzílico 3-O-benzil), 4,72 (d, $\left.1 \mathrm{H}, J_{1-2}=4,0 \mathrm{~Hz}, \mathrm{H}-1\right), 4,68\left(\mathrm{~d}, 1 \mathrm{H}, J_{\text {geminal }}=\right.$ $12,4 \mathrm{~Hz}, \mathrm{H}$ benzílico 2-O-benzil), 4,58 (d, $1 \mathrm{H}, J_{\text {geminal }}=11,6 \mathrm{~Hz}, \mathrm{H}$ benzílico 3-O-benzil), 4,16-4,15 (m, $1 \mathrm{H}, \mathrm{H}-5), 4,06$ (dd, $1 \mathrm{H}, J_{2-3}$ $\left.=10,0 \mathrm{~Hz}, J_{1-2}=4,0 \mathrm{~Hz}, \mathrm{H}-3\right), 4,01-3,91$ (m, $\left.2 \mathrm{H}, \mathrm{H}-7\right), 3,60-3,49$ (m, 3 H, H-2 e H-6), 3,42 (s, 3 H, H metoxila), 2, 19 (s, 3 H, H-18). $\mathrm{RMN}{ }^{13} \mathrm{C}\left(100 \mathrm{MHz}, \mathrm{CDCl}_{3}\right), \delta: 168,4,167,8$ (C-10 e C-17), 138,4, 138,2 (C ipso dos grupos benzila), 137,6, 135,8 (C-11 e C-13), 134,4 (C-8), 129,4 (C-15), 128,4, 128,3, 128,1, 128,0, 127,8, 127,5 (C aromáticos), 123,0, 122,4 (C-14 e C-16), 118,5 (C-12), 117,3 (C-9), 98,7 (C-1), 76,5 (C-3), 75,6 (C-2), 73,6 (C benzílico 2-O-benzil), 72,5 (C-7), 71,6 (C benzílico 3-O-benzil), 69,7 (C-6), 68,0 (C-5), 55,5 (C metoxila), 48,9 (C-4), 24,6 (C-18).

Pelo tratamento da iodoacetamida 20 pelo mesmo procedimento descrito para a amida $\mathbf{1 3}$ foi obtido o produto de hidrogenólise $\mathbf{3 8}$ (11,7 mg; $18 \mathrm{mmol} ; 10 \%)$. Sólido branco. PF: 55,0-58,2 ${ }^{\circ} \mathrm{C} .[\alpha]_{\mathrm{D}}$ $+32,1\left(c 1,10, \mathrm{CHCl}_{3}\right)$. IV $\left(\mathrm{cm}^{-1}\right.$, filme, $\left.v_{\mathrm{ma}}\right): 3304,3062,3029,2919$, 2851, 1646, 1610, 1496, 1453, 1095. RMN ${ }^{1} \mathrm{H}\left(400 \mathrm{MHz}, \mathrm{CDCl}_{3}\right)$, $\delta: 7,86\left(\mathrm{~d}, 1 \mathrm{H}, J_{\text {orto }}=7,6 \mathrm{~Hz}, \mathrm{H}-14\right), 7,65$ (s, $\left.1 \mathrm{H}, \mathrm{H}-12\right), 7,41-7,15$ $\left(\mathrm{m}, 18 \mathrm{H}, \mathrm{H}\right.$ aromáticos e $\mathrm{H}-\mathrm{N}$ acetamida), 6,52 (d, $1 \mathrm{H}, J_{8-9}=16,0$ $\mathrm{Hz}, \mathrm{H}-9), 6,31$ (d, $1 \mathrm{H}, J_{\mathrm{NH}-4}=9,6 \mathrm{~Hz}, \mathrm{H}-\mathrm{N}$ benzamida), 6,19 (td, 1 $\left.\mathrm{H}, J_{8-9}=16,0 \mathrm{~Hz}, J_{7-8}=6,1 \mathrm{~Hz}, \mathrm{H}-8\right), 4,98\left(\mathrm{ddd}, 1 \mathrm{H}, J_{\mathrm{NH}-4}=9,6 \mathrm{~Hz}\right.$, $\left.J_{3-4}=4,5 \mathrm{~Hz}, J_{4-5}=1,1 \mathrm{~Hz}, \mathrm{H}-4\right), 4,89\left(\mathrm{~d}, 1 \mathrm{H}, J_{\text {geminal }}=11,2 \mathrm{~Hz}, \mathrm{H}\right.$ benzílico 3-O-benzil), 4,85 (d, $1 \mathrm{H}, J_{\text {geminal }}=12,2 \mathrm{~Hz}, \mathrm{H}$ benzílico 2- $O$-benzil), 4,73 (d, $\left.1 \mathrm{H}, J_{1-2}=3,6 \mathrm{~Hz}, \mathrm{H}-1\right), 4,68\left(\mathrm{~d}, 1 \mathrm{H}, J_{\text {geminal }}=\right.$ $12,2 \mathrm{~Hz}, \mathrm{H}$ benzílico 2- $O$-benzil), 4,58 (d, $1 \mathrm{H}, J_{\text {geminal }}=11,2 \mathrm{~Hz}, \mathrm{H}$ benzílico 3-O-benzil), 4,20-4,16 (m, $1 \mathrm{H}, \mathrm{H}-5), 4,12$ (ddd, $2 \mathrm{H}, J$ $\left.=10,8 \mathrm{~Hz}, J_{7-8}=6,1 \mathrm{~Hz}, J_{7-9}=0,9 \mathrm{~Hz}, \mathrm{H}-7\right), 4,06\left(\mathrm{dd}, 1 \mathrm{H}, J_{2-3}=9,8\right.$ $\left.\mathrm{Hz}, J_{3-4}=4,5 \mathrm{~Hz}, \mathrm{H}-3\right), 3,66-3,54$ (m, $\left.3 \mathrm{H}, \mathrm{H}-2 \mathrm{e} \mathrm{H}-6\right), 3,43$ (s, $3 \mathrm{H}$, $\mathrm{H}$ metoxila), 2,15 (s, $3 \mathrm{H}, \mathrm{H}-18) . \mathrm{RMN}{ }^{13} \mathrm{C}\left(100 \mathrm{MHz}, \mathrm{CDCl}_{3}\right), \delta$ : 168,3, 167,7 (C-10 e C-17), 138,4, 138,31, 138,26 (C ipso dos grupos benzila e C-11, C-13 ou C ipso do grupo cinamila), 136,6, 135,5 (C11, C-13 ou C ipso do grupo cinamila), 132,8 (C-9), 129,4 (C-15), 128,5, 128,4, 128,3, 128,11, 128,06, 127,8, 126,5 (C aromáticos), 125,7 (C-8), 123,0, 122,4 (C-14 e C-16), 118,5 (C-12), 98,8 (C-1), 76,5 (C-3), 75,5 (C-2), 73,6 (C benzílico 2-O-benzil), 72,2 (C-7), 71,6 (C benzílico 3-O-benzil), 69,6 (C-6), 67,9 (C-5), 55,5 (C metoxila), 48,9 (C-4), 24,6 (C-18).

\section{AGRADECIMENTOS}

Ao CNPq pelo suporte financeiro, pelas bolsas de produtividade em pesquisa (M. A. F. Prado e R. J. Alves) e pelas bolsas de iniciação científica (M. C. Pires e C. G. Queiroga). À FAPEMIG pela bolsa de doutorado (D. H. S. Leal). 


\section{REFERÊNCIAS}

1. Ramaseshan, M.; Robitaille, M.; Ellingboe, J. W.; Dory, Y. L.; Deslongchamps, P.; Tetrahedron Lett. 2000, 41, 4737.

2. Blankenstein, J.; Zhu, J. P.; Eur. J. Org. Chem. 2005, 1949.

3. Wessjohann, L. A.; Ruijter, E.; Garcia-Rivera, D.; Brandt, W.; Mol. Diversity 2005, 9, 171.

4. Nicolaou, K. C.; Tetrahedron 1977, 33, 683.

5. Beckwith, A. L. J.; Gara, W.; J. Chem. Soc, Perkin Trans. 2 1975, 593.

6. Porter, N. A.; Magnin, D. R.; Wright, B. T.; J. Am. Chem. Soc. 1986, $108,2787$.

7. Porter, N. A.; Chang, V. H. T.; J. Am. Chem. Soc. 1987, 109, 4976.

8. Porter, N. A.; Chang, V. H. T.; Magnin, D. R.; Wright, B. T.; J. Am. Chem. Soc. 1988, 110, 3554.

9. Giese, B.; Radicals in Organic Synthesis: Formation of carbon-carbon bonds, Pergamon Pres: Oxford, 1988.

10. Ferrier, R. J.; Middleton, S.; Chem. Rev. 1993, 93, 2779.

11. Perkins, M. J.; Radical Chemistry, Ellis Horwood: New York, 1995.

12. Beckwith, A. L. J.; Drok, K.; Maillard, B.; Degueil-Castaing, M.; Philippon, A.; Chem. Commun. 1997, 499.

13. Ishibashi, H.; Kawanami, H.; Ikeda; M.; J. Chem. Soc., Perkin Trans. 1 1997, 817.

14. Bowman, W. R.; Mann, M.; Parr, J.; J. Chem. Soc., Perkin Trans. 12000 , 2991.

15. Brunton, S. A.; Jones, K.; J. Chem. Soc., Perkin Trans. 1 2000, 763.

16. Escolano, C.; Jones, K.; Tetrahedron Lett. 2000, 41, 8951.

17. Ishibashi, H.; Kato, I.; Takeda, Y.; Kogure, M.; Tamura, O.; Chem. Commun. 2000, 1527.

18. Clayden, J.; Greeves, N.; Warren, S.; Wothers, P.; Organic chemistry, Oxford University: New York, 2001.

19. Allin, S. M.; Barton, W. R. S.; Bowman, W. R.; McInally, T.; Tetrahedron Lett. 2002, 43, 4191.

20. Curran, D. P.; Yang, F.; Cheong, J.; J. Am. Chem. Soc. 2002, 124, 14993.

21. Ishibashi, H.; Sato, T.; Ikeda, M.; Synthesis 2002, 695.

22. Jones, K.; Wilkinson, J.; J. Chem. Soc., Chem. Commun. 1992, 1767.

23. Jessop, C. N.; Parsons, A. F.; Routledge, A.; Irvine, D.; Tetrahedron Lett. 2003, 44, 479 .

24. Prado, M. A. F.; Alves, R. J.; Souza Filho, J. D.; Alves, R. B.; Pedrosa, M. T. C.; Prado, R. F.; Faraco, A. A. G.; J. Chem. Soc., Perkin Trans. 12000, 1853.

25. Binatti, I.; Prado, M. A. F.; Alves, R. J.; Souza-Filho, J. D.; J. Braz. Chem. Soc. 2002, 13, 570 .

26. Faraco, A. A. G.; Prado, M. A. F.; Alves, R. J.; Souza Filho, J. D.; Alves, R. B.; Faraco, R. F. P.; Synth. Commun. 2003, 33, 463.
27. Oliveira, R. B.; Prado, M. A. F.; Alves, R. J.; Souza Filho, J. D.; J. Braz. Chem. Soc. 2003, 14, 442.

28. Oliveira, R. B.; Prado, M. A. F.; Alves, R. J.; Souza Filho, J. D.; Meurer, E. C.; Eberlin, M. N.; Santos, L. S.; Tetrahedron 2004, 60, 9901.

29. Faraco, A. A. G.; Prado, M. A. F.; Alves, R. J.; Souza Filho, J. D.; Alves, R. B.; Prado, R. F.; Meurer, E. C.; Eberlin, M. N.; Tetrahedron Lett. 2004, 45, 3317.

30. Binatti, I.; Alves, R. B.; Prado, M. A. F.; Alves, R. J.; Souza-Filho, J. D.; Dias, D. F.; Quim. Nova 2005, 28, 1023.

31. Dias, D. F.; Prado, M. A. F.; Pinto, G. D.; Alves, R. J.; Alves, R. B.; Souza Filho, J. D.; Quim. Nova 2006, 29, 444.

32. Oliveira, M. T.; Prado, M. A. F.; Alves, R. B.; Cesar, A.; Alves, R. J.; Queiroga, C. G.; Santos, L. S.; Eberlin, M. N.; J. Braz. Chem. Soc. 2007, 18,364 .

33. Faraco, R. F. P.; Pires, M. C.; Rocha, A. P. C.; Prado, M. A. F.; Quim. Nova 2008, 31, 1499.

34. Taniguchi, T.; Yonei, D.; Sasaki, M.; Tamura, O.; Ishibashi, H.; Tetrahedron 2008, 64, 2634.

35. Liu, L. Chen, Q.; Wu, Y. D.; Li, C.; J. Org. Chem. 2005, 70, 1539.

36. Ghosh, K.; Ghatak, U. R.; Tetrahedron Lett. 1995, 36, 4897.

38. Harrowven, D. C. L'Helias, N.; Moseley, J. D.; Blumire, N. J.; Flanagan, S. R.; Chem. Commun. 2003, 2658.

39. Srikanth, G. S. C.; Castle, S. L.; Tetrahedron 2005, 61, 10377.

40. Lichtenthaler, F. W.; Heidel, P. H.; J. Org. Chem. 1974, 39, 1457.

41. Hall, D. M.; Carbohydr. Res. 1980, 86, 158.

42. Pietraszkiewicz, M.; Jurczak, J.; Tetrahedron 1984, 40, 2967.

43. Bell, D. J.; Lorber, J.; J. Chem. Soc. 1940, 453.

44. Figueiredo, R. C.; Dissertação de Mestrado, Universidade Federal de Minas Gerais, Brasil, 2000.

45. Umezawa, S.; Nishimura, Y.; Hata, Y.; Tsuchiya, T.; J. Antibiot. 1974, 27, 722 .

46. Dufour, M.; Gramain, J. C.; Husson, H. P.; Sinibaldi, M. E.; Troyn, Y.; Synth. Commun. 1992, 22, 189.

47. Beak, P.; Musick, T. J.; Chen, C.; J. Am. Chem. Soc. 1988, 110, 3538.

48. Vogel, A. I.; Química orgânica, Ao Livro Técnico: Rio de Janeiro, 1982, vol. 2.

49. Martin, T. A.; Comer, W. T.; J. Med. Chem. 1985, 28, 910.

50. Leone-Bay, A.; Timony, P. E.; Synthetic Comm. 1988, 18, 1637.

51. Marinovic, N. N.; Ramanathan, H.; Tetrahedron Lett. 1983, 24, 1871.

52. Curran, D. P.; Tamine, J.; J. Org. Chem. 1991, 56, 2746.

53. Leal, D. H. S.; Cesar, A.; trabalho não publicado.

54. Silverstein, R. M.; Webster, F. X.; Kiemle, D. J.; Spectrometric Identification of Organic Compounds, $7^{\text {th }}$ ed, Wiley: New York, 2005, cap. 3. 